\title{
GCU
}

Glasgow Caledonian

University

University for the Common Good

\section{Environmental and behavioural interventions for reducing physical activity limitation and preventing falls in older people with visual impairment}

E, Jian-Yu; Li, Tianjing; McInally, Lianne; Thomson, Katie; Shahani, Uma; Gray, Lyle; Howe, Tracey E.; Skelton, Dawn A.

Published in:

Cochrane Database of Systematic Reviews

DOI:

10.1002/14651858.CD009233.pub3

Publication date:

2020

Document Version

Publisher's PDF, also known as Version of record

Link to publication in ResearchOnline

Citation for published version (Harvard):

E, J-Y, Li, T, Mclnally, L, Thomson, K, Shahani, U, Gray, L, Howe, TE \& Skelton, DA 2020, 'Environmental and behavioural interventions for reducing physical activity limitation and preventing falls in older people with visual impairment', Cochrane Database of Systematic Reviews, vol. 2020, no. 9, CD009233, pp. CD009233.

https://doi.org/10.1002/14651858.CD009233.pub3

\section{General rights}

Copyright and moral rights for the publications made accessible in the public portal are retained by the authors and/or other copyright owners and it is a condition of accessing publications that users recognise and abide by the legal requirements associated with these rights.

Take down policy

If you believe that this document breaches copyright please view our takedown policy at https://edshare.gcu.ac.uk/id/eprint/5179 for details

of how to contact us. 


\section{Environmental and behavioural interventions for reducing physical activity limitation and preventing falls in older people with visual impairment (Review)}

E JY, Li T, McInally L, Thomson K, Shahani U, Gray L, Howe TE, Skelton DA

E J-Y, Li T, McInally L, Thomson K, Shahani U, Gray L, Howe TE, Skelton DA.

Environmental and behavioural interventions for reducing physical activity limitation and preventing falls in older people with visual impairment.

Cochrane Database of Systematic Reviews 2020, Issue 9. Art. No.: CD009233.

DOI: 10.1002/14651858.CD009233.pub3. 
HEADER 1

ABSTRACT

PLAIN LANGUAGE SUMMARY

SUMMARY OF FINDINGS

BACKGROUND

OBJECTIVES

METHODS

RESULTS

Figure 1.

Figure 2.

Figure 3.

Figure 4.

Figure 5.

Figure 6.

Figure 7.

Figure 8.

DISCUSSION

AUTHORS' CONCLUSIONS

ACKNOWLEDGEMENTS

REFERENCES

CHARACTERISTICS OF STUDIES

APPENDICES

WHAT'S NEW

HISTORY

CONTRIBUTIONS OF AUTHORS

DECLARATIONS OF INTEREST

SOURCES OF SUPPORT

DIFFERENCES BETWEEN PROTOCOL AND REVIEW

NOTES

INDEX TERMS

\section{TABLE OF CONTENTS}

1

2

4

7

8

8

10

11

13

15

15

16

16 
[Intervention Review]

\title{
Environmental and behavioural interventions for reducing physical activity limitation and preventing falls in older people with visual impairment
}

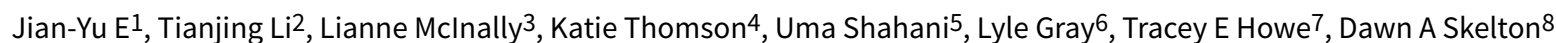

1Department of Epidemiology, Johns Hopkins Bloomberg School of Public Health, Baltimore, Maryland, USA. 2Department of Ophthalmology, University of Colorado Denver Anschutz Medical Campus, Aurora, CO, USA. ${ }^{3}$ Lanarkshire Falls Service, Lanarkshire, UK. ${ }^{4}$ Glasgow Caledonian University, Glasgow, UK. ${ }^{5}$ Department of Visual Sciences, Glasgow Caledonian University, Glasgow, UK. 6 Life Sceince, Glasgow Caledonian University, Glasgow, UK. ${ }^{7}$ City of Glasgow College, Glasgow, UK. ${ }^{8}$ School of Health \& Life Sciences, Institute of Applied Health Research, Glasgow Caledonian University, Glasgow, UK

Contact address: Dawn A Skelton, dawn.skelton@gcu.ac.uk, DAWNASKELTON@GMAIL.COM.

Editorial group: Cochrane Eyes and Vision Group.

Publication status and date: New search for studies and content updated (conclusions changed), published in Issue 9, 2020.

Citation: E J-Y, Li T, McInally L, Thomson K, Shahani U, Gray L, Howe TE, Skelton DA. Environmental and behavioural interventions for reducing physical activity limitation and preventing falls in older people with visual impairment. Cochrane Database of Systematic Reviews 2020, Issue 9. Art. No.: CD009233. DOI: 10.1002/14651858.CD009233.pub3.

Copyright ( 2020 The Cochrane Collaboration. Published by John Wiley \& Sons, Ltd.

\section{A B S T R A C T}

\section{Background}

Impairment of vision is associated with a decrease in activities of daily living. Avoidance of physical activity in older adults with visual impairment can lead to functional decline and is an important risk factor for falls. The rate of falls and fractures is higher in older people with visual impairment than in age-matched visually normal older people. Possible interventions to reduce activity restriction and prevent falls include environmental and behavioral interventions.

\section{Objectives}

We aimed to assess the effectiveness and safety of environmental and behavioral interventions in reducing physical activity limitation, preventing falls and improving quality of life amongst visually impaired older people.

\section{Search methods}

We searched CENTRAL (including the Cochrane Eyes and Vision Trials Register) (Issue 2, 2020), Ovid MEDLINE, Embase and eight other databases to 4 February 2020, with no language restrictions.

\section{Selection criteria}

Eligible studies were randomized controlled trials (RCTs) and quasi-randomized controlled trials (Q-RCTs) that compared environmental interventions, behavioral interventions or both, versus control (usual care or no intervention); or that compared different types of environmental or behavioral interventions. Eligible study populations were older people (aged 60 and over) with irreversible visual impairment, living in their own homes or in residential settings. To be eligible for inclusion, studies must have included a measure of physical activity or falls, the two primary outcomes of interest. Secondary outcomes included fear of falling, and quality of life.

\section{Data collection and analysis}

We used standard Cochrane methods. We assessed the certainty of the evidence using the GRADE approach. 


\section{Main results}

We included six RCTs (686 participants) conducted in five countries (Australia, Hungary, New Zealand, UK, US) with follow-up periods ranging from two to 12 months. Participants in these trials included older adults (mean age 80 years) and were mostly female (69\%), with visual impairments of varying severity and underlying causes. Participants mostly lived in their homes and were physically independent. We classified all trials as having high risk of bias for masking of participants, and three trials as having high or unclear risk of bias for all other domains. The included trials evaluated various intervention strategies (e.g. an exercise program versus home safety modifications). Heterogeneity of study characteristics, including interventions and outcomes, (e.g. different fall measures), precluded any meta-analysis.

Two trials compared the home safety modification by occupational therapists versus social/home visits. One trial (28 participants) reported physical activity at six months and showed no evidence of a difference in mean estimates between groups (step counts: mean difference $(M D)=321,95 \%$ confidence interval $(\mathrm{Cl})-1981$ to 2623 ; average walking time (minutes): $\mathrm{MD} 1.70,95 \% \mathrm{Cl}-24.03$ to 27.43 ; telephone questionnaire for self-reported physical activity: MD -3.68 scores, $95 \% \mathrm{Cl}-20.6$ to 13.24 ; low-certainty of evidence for each outcome). Two trials reported the proportion of participants who fell at six months (risk ratio (RR) $0.76,95 \% \mathrm{Cl} 0.38$ to $1.51 ; 28$ participants) and 12 months (RR $0.59,95 \% \mathrm{Cl} 0.43$ to $0.80,196$ participants) with low-certainty of evidence for each outcome. One trial (28 participants) reported fear of falling at six months, using the Short Falls Efficacy Scale-International, and found no evidence of a difference in mean estimates between groups (MD 2.55 scores, $95 \% \mathrm{Cl}-0.51$ to 5.61 ; low-certainty of evidence). This trial also reported quality of life at six months using 12 -Item Short Form Health Survey, and showed no evidence of a difference in mean estimates between groups (MD -3.14 scores, $95 \% \mathrm{Cl}-10.86$ to 4.58; low-certainty of evidence).

Five trials compared a behavioral intervention (exercise) versus usual activity or social/home visits. One trial (59 participants) assessed selfreported physical activity at six months and showed no evidence of a difference between groups (MD 9.10 scores, 95\% Cl - 13.85 to 32.5 ; lowcertainty of evidence). Three trials investigated different fall measures at six or 12 months, and found no evidence of a difference in effect estimates (RRs for proportion of fallers ranged from 0.54 ( $95 \% \mathrm{Cl} 0.29$ to $1.01 ; 41$ participants); to 0.93 ( $95 \% \mathrm{Cl} 0.61$ to $1.39 ; 120$ participants); low-certainty of evidence for each outcome). Three trials assessed the fear of falling using Short Falls Efficacy Scale-International or the Illinois Fear of Falling Measure from two to 12 months, and found no evidence of a difference in mean estimates between groups (the estimates ranged from -0.88 score $(95 \% \mathrm{Cl}-2.72$ to $0.96,114$ participants) to 1.00 score $(95 \% \mathrm{Cl}-0.13$ to 2.13 ; 59 participants); low-certainty of evidence). One trial (59 participants) assessed the European Quality of Life scale at six months (MD -0.15 score, $95 \% \mathrm{Cl}-0.29$ to -0.01 ), and found no evidence of a clinical difference between groups (low-certainty of evidence).

\section{Authors' conclusions}

There is no evidence of effect for most of the environmental or behavioral interventions studied for reducing physical activity limitation and preventing falls in visually impaired older people. The certainty of evidence is generally low due to poor methodological quality and heterogeneous outcome measurements.

Researchers should form a consensus to adopt standard ways of measuring physical activity and falls reliably in older people with visual impairments. Fall prevention trials should plan to use objectively measured or self-reported physical activity as outcome measures of reduced activity limitation. Future research should evaluate the acceptability and applicability of interventions, and use validated questionnaires to assess the adherence to rehabilitative strategies and performance during activities of daily living.

\section{PLAIN LANGUAGE SUMMARY}

\section{How effective are home improvements and other strategies designed to help older people with low vision move around safely?}

\section{Why this question is important}

Low vision that cannot be corrected by standard glasses or any medical treatment is called visual impairment. It is common for people to become visually impaired in later life. Older people with visual impairment tend to avoid physical activity; for example, they might walk fewer steps in a day than people who are not visually impaired. They are also more likely to experience anxiety or depression, or to sustain an injury through falling.

Changes to a person's environment or behavior can be made to help older, visually impaired people move safely around - inside and outside - their homes. These include adaptations to the home to enable safe movement around it, or improvement to the person's balance through exercise.

To find out if strategies designed to help people move around safely are effective for older people with visual impairment, a team of Cochrane researchers reviewed the evidence from research studies. Specifically, we wanted to know if these strategies:

- increase physical activity;

- reduce risk of falling;

- decrease fear of falling;

- increase quality of life.

\section{How we identified and assessed the evidence}


First, we searched for all relevant studies in the medical literature. We then compared the results, and summarized the evidence from all the studies. Finally, we assessed how certain the evidence was. We considered factors such as the way studies were conducted, study sizes, and consistency of findings across studies. Based on our assessments, we categorized the evidence as being of very low-, low-, moderateor high-certainty.

\section{What we found}

We found six studies in a total of 686 older people with visual impairment who were followed for between two and 12 months. People who took part in the studies were aged 80 years on average, and they lived either at home or in a residential setting.

Five studies compared changes to the home made by occupational therapists versus home visits from research staff or volunteers. The evidence from these studies suggests that six months after they were made, changes to the home may make little to no difference to physical activity, fear of falling or quality of life (low-certainty evidence). Changes to the home may make little to no difference to people's risk of falling six months after they were made, but may slightly reduce risk of falling after one year (low-certainty evidence).

Six studies compared exercise versus usual activities or home visits. The evidence from these studies suggests that exercise may make little to no difference to physical activity, risk of falling, fear of falling or quality of life after six months (low-certainty evidence).

\section{What this means}

Current evidence suggests that strategies designed to help people move around safely may not increase physical activity, reduce fear of falling or improve the quality of life of older people with visual impairment. Changes to a home to improve how safe it is to move around in it may slightly reduce risk of falling after one year, but not after six months.

The certainty of the evidence is low. Further research is likely to change the findings of this review. Future studies that investigate which strategies are acceptable to people and why, and that measure changes in physical activity and falls using robust research methods, will help to reduce uncertainty in this field.

\section{How-up-to date is this review?}

The evidence in this Cochrane Review is current to 4 February 2020. 


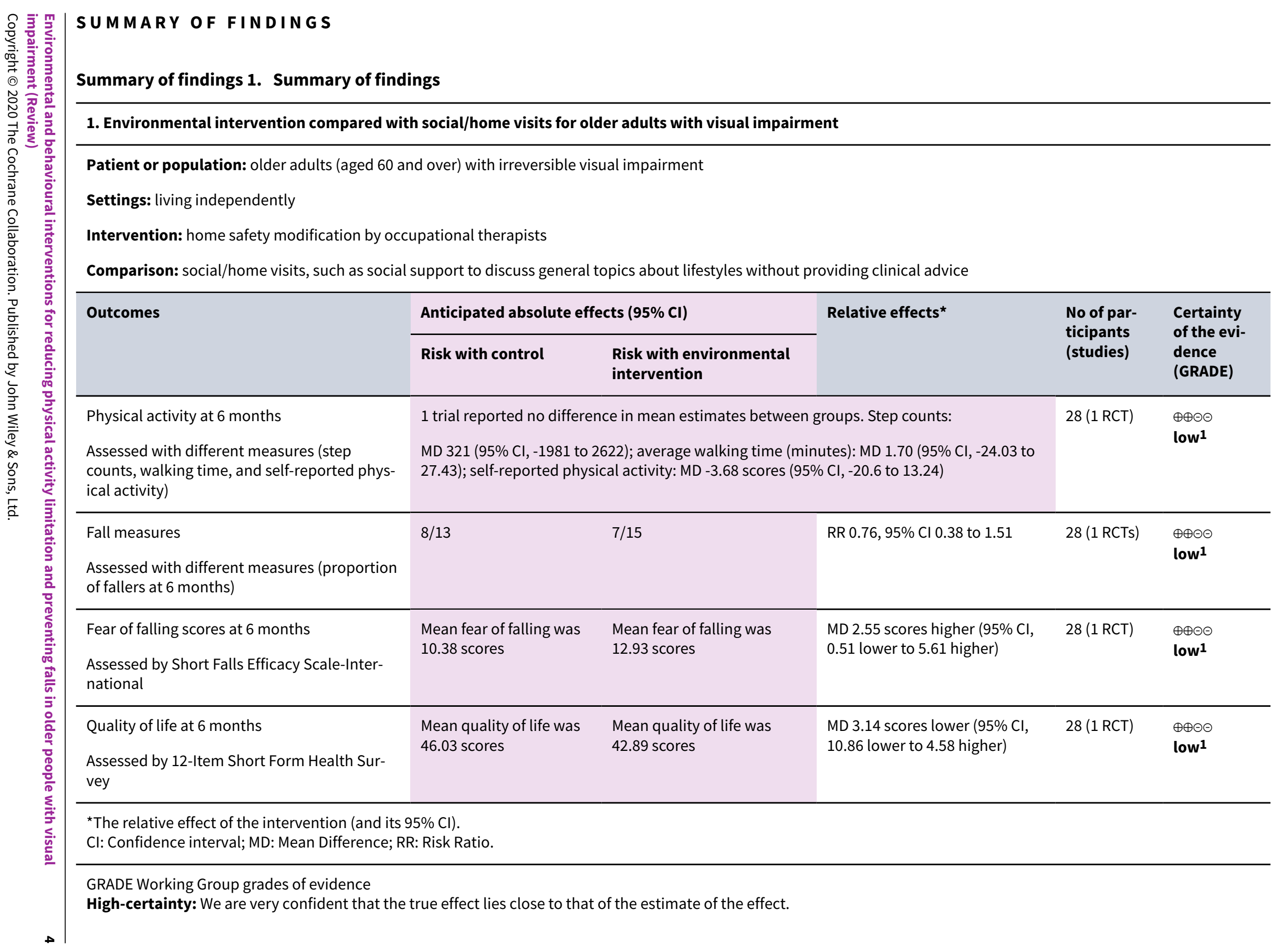




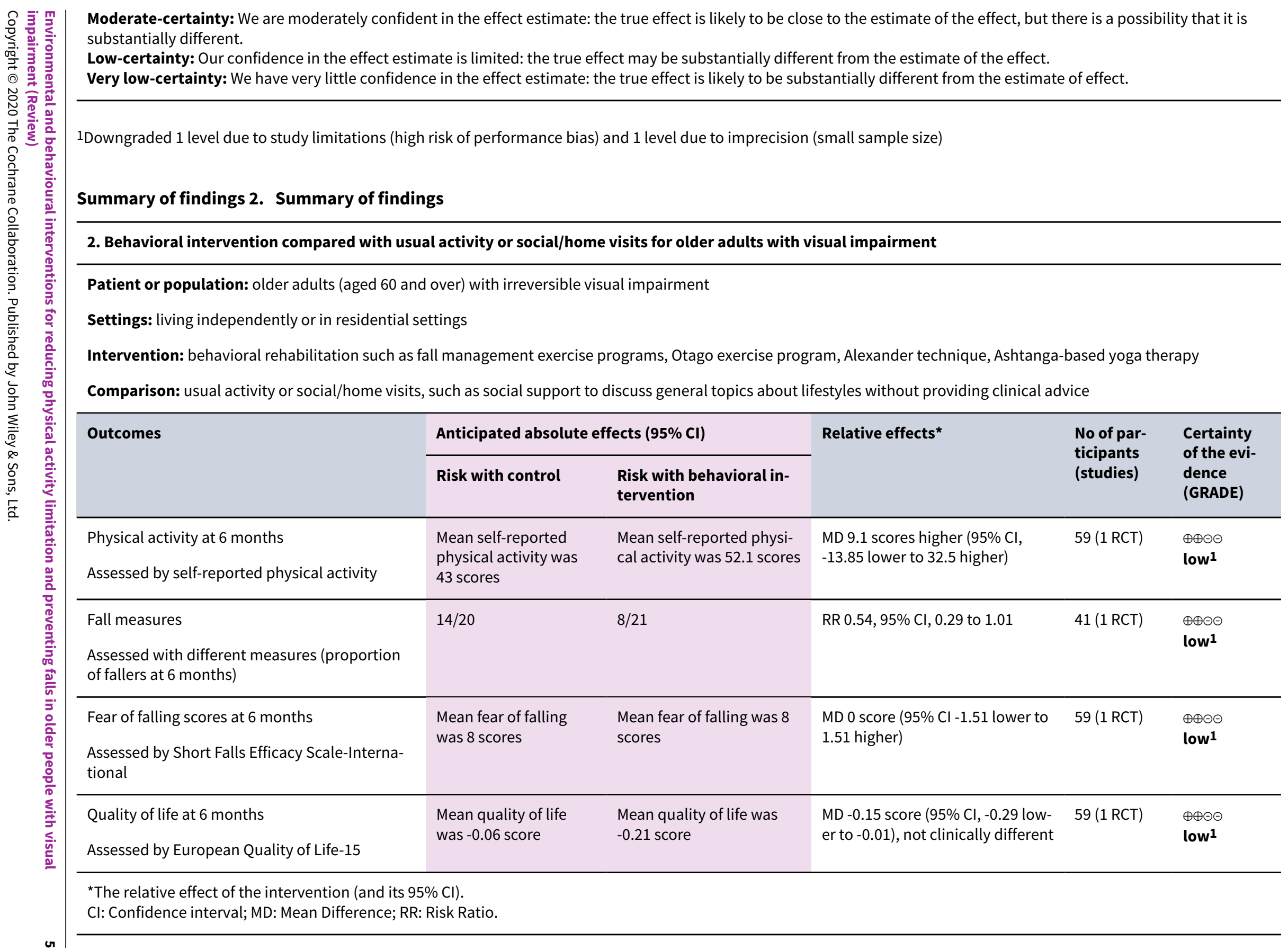




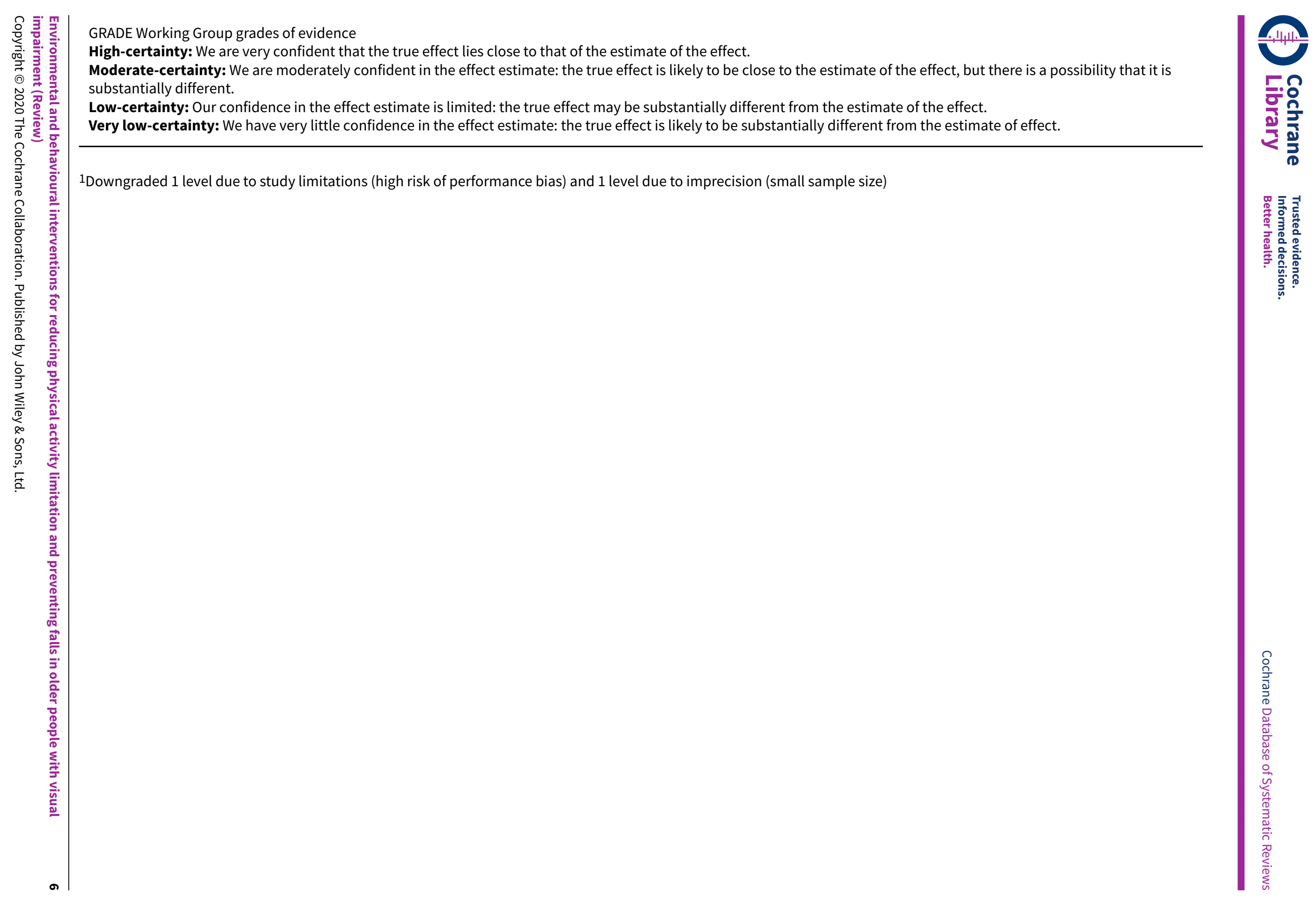




\section{B A C K G R O U N D}

The prevalence of visual impairment is estimated by the World Health Organization (WHO) to be 285 million worldwide (Pascolini 2012). One in eight people in the UK over the age of 75 and one in three over the age of 90 live with significant sight loss (Evans 2002). Older adults with visual impairment are more likely to be physically dependent (Klein 2003), have higher risk of moving into residential settings (e.g. nursing homes and long-term care settings) (Ivers 1998; Tinetti 1998), and have poorer functions in daily living (Brouwer 2008; E 2020a; Salive 1994) than normally sighted peers. Vision deterioration is also associated with adverse health consequences among older people, especially with regard to activity limitations (Ong 2018). These include decreases in leisure activities, Instrumental Activities of Daily Living performance and social function, Activities of Daily Living and compromised mobility. The International Classification of Functioning defines activity as "the nature and extent of performance of a function by a person" and activity limitations as "problems of the performance of activities in nature, duration, and quality" (WHO 2001). Avoidance or lack of physical activity by older adults with low vision may have implications for other health problems (WHO 2010), such that social isolation, anxiety and depression should be noted by ophthalmologists and others treating these populations (Berger 2012). Challenging environments, struggling to obtain transportation, feelings of vulnerability, having decreased energy, and lacking assertiveness were all identified in interviews with older visually impaired adults as reasons for not being physically active and not feeling competent in such activities (Berger 2012).

Visual impairment is associated with an increase in the incidence of falls, hip fractures, and depression (Crews 2004). Avoidance of physical activity because of fear of falling is common among older people at risk of falling (Yardley 2002; E 2020b). Indeed, the rate of falls in older people with visual impairment is 1.7 times higher than in age-matched visually normal populations; the rate for hip fracture is 1.3 to 1.9 times higher (Legood 2002). One study reported that activity restriction was present in $45 \%$ of those with visual impairment, compared with only $24 \%$ in those without visual impairment, who had experienced an injurious fall (Murphy 2002). Those with visual impairment were also more likely to admit to fear of falling (44\%) even without a fall history. One study has shown that perceived interference of vision loss on goal-directed behavior and expected activities has greater influence on distress and is subsequently predictive of disability, in comparison with objective symptoms (visual acuity) (Dreer 2008). It therefore seems likely that for people with visual impairment, the mechanism underpinning previous trials of maintaining physical activity and preventing falls, particularly with respect to environmental components, behavioral components or both, will be different from that of the general population.

\section{Description of the condition}

Visual impairment is defined as best-corrected visual acuity of the better eye less than $0.3 \log$ MAR (Log of the Minimum Angle of Resolution) units or visual field defects within 20 degrees of fixation. Blindness is defined as visual acuity less than 0.05 logMAR units or visual field defects within 10 degrees of fixation (WHO 2019). A working definition of visual impairment is low vision that cannot be corrected by standard glasses or by medical or surgical intervention. The top five conditions leading to visual impairment in the US are diabetic retinopathy, age-related macular degeneration, cataract, glaucoma, and eye injury or trauma (Welp 2016). Age-related macular degeneration and diabetic retinopathy are the most common causes of blindness in Americans over 65 years of age (Pelletier 2016).

\section{Description of the intervention}

Environmental interventions include any targeted, intentional improvement to the usually indoor physical environment, with the aim of reducing symptoms or improving well-being (Preedy 2009). In the case of visual impairment, this may incorporate adaptations and modifications to an individual's physical environment (usually their home) as the result of a formal environmental assessment that identified potential hazards or restrictions. The aim of the environmental intervention is to enhance the individual's ability to perform daily living tasks safely and independently, facilitating their safe mobility and improving confidence. Examples of environmental interventions for individuals with visual impairment include the removal of rugs, increased lighting in hallways and applying contrasting stripes on stairs.

Behavioral interventions include the systematic implementation of procedures that result in lasting positive changes in an individual's behavior (Markowitz 2006a). For people with visual impairment, these interventions may include teaching of adaptive strategies to enhance changes in an individual's behavior when navigating and interacting with their environment (Markowitz 2006b) and orientation and mobility training (Virgili 2010), among other interventions. Orientation and mobility training aims to teach visually impaired people how to ambulate and navigate the environment safely and independently. It may contribute to reducing activity limitations and enhancing societal participation (Zijlstra 2009).

Occupational therapists have the expertise to assess, devise and implement rehabilitation plans which incorporate both types of interventions: an occupational therapy approach encompasses both environmental change and the interaction of the individual with their environment, their actions and their behavioral adaptations at home and in the community. This dynamic relationship between the person, their behavior and the environment has been described elsewhere (Clemson 2003; Markowitz 2006b). Many environmental risk assessments, and some environmental modifications, are undertaken by other professionals but the relationship of the person, their behavior and their environment may not be evaluated comprehensively.

\section{How the intervention might work}

Through changes in the home environment and behavioral strategies, persons with visual impairment may feel more confident that they can navigate their environment safely, thereby reducing concerns about their safety and fear of falling. This improved muscle strength and balance may lead to greater mobility and habitual physical activity, and lower risk of falling. Although increased physical activity can increase exposure to falls, studies with exercise interventions which focus on stability and strength have been shown not to increase risk of falls (Mihailovic 2017; Ong 2018; Ramulu 2009).

\section{Why it is important to do this review}

Avoidance of activity is more common and the risk of falls is greater in older people with visual impairment than those with 
normal vision (Legood 2002). Although there exists a suite of Cochrane reviews on falls prevention in older people living in the community (Hopewell 2018; Sherrington 2019), in residential settings (Cameron 2018), as well as on exercise to reduce fear of falling (Kendrick 2014), they do not include all studies in participants with specific conditions (e.g. those with visual impairment), or do not have sub-analyses on those studies in older people with visual impairment.

The physiological, psychological, functional and societal benefits of regular physical activity amongst older people (WHO 2007) are irrefutable. Interventions that improve habitual physical activity in visually impaired older people are vital to promoting public health. Trials that have adopted a holistic, participant-centered approach (such as environment modification led by occupational therapies) have shown reductions in falls both within and outside the home), whereas trials that have considered removal of home hazards only did not reduce the incidence of falls, even within the home. One potential mechanism for falls reduction could be that the visually impaired individual actually restricts activity more as a result of the intervention, which in the short term could reduce exposure to falls risk. There is therefore a need to further unpick the mechanism of reduction in falls as well as maintaining or increasing physical activity in visually impaired older people. Since trials have rarely considered activity restriction alongside falls as an outcome measure, the effectiveness and safety of environmental and behavioral interventions remain unclear.

The original version of this review (Skelton 2013) found no eligible studies. The topic of maintaining physical activity and preventing falls in older adults with vision impairment remains highly relevant to patients, care givers, providers, insures, and policy makers. Therefore, an update is needed to examine the recent evidence with regard to the effect of environmental or behavioral interventions for reducing physical activity limitation and preventing falls in visually impaired older people.

\section{O B J E C T I VES}

The objective of this review was to assess the effectiveness and safety of environmental and behavioral interventions in reducing activity limitation, preventing falls, and improving quality of life amongst visually impaired older people.

\section{METHODS}

\section{Criteria for considering studies for this review}

\section{Types of studies}

Eligible studies were randomized controlled trials (RCTs) and quasi-randomized controlled trials (Q-RCTs) that compared environmental interventions, behavioral interventions or both, versus control (placebo control or usual care or no intervention); or that compared different types of environmental or behavioral interventions. We did not include studies that report only quality of life (as opposed to limitations in mobility and quality of life) so as to avoid overlap with another Cochrane Review (van Nispen 2020).

\section{Types of participants}

We included trials with the following participants.
- Older people (aged 60 and over) with irreversible visual impairment including, but not limited to, low visual acuity, poor contrast sensitivity, poor depth perception and reduced visual field.

- Older people with irreversible visual impairment and other multiple disabilities, such as hearing loss, neurological or musculoskeletal disease or cognitive impairments.

- Older people living independently and those living in residential settings.

\section{Types of interventions}

Environmental interventions, behavioral interventions or both, including but not limited to visual rehabilitation (e.g. low vision devices), removal of home hazards, home safety modifications, provision of adaptive or assistive equipment, advice on behavioral changes to improve safety in activities of daily living, cognitive behavioral therapies, or other behavioral therapies.

For each study included, we aimed to record the professional training of the person delivering the interventions. The types of interventions were also rated on the intensity of the intervention, based on previously published criteria (evaluation of risk of person and environment; validated assessment tools; formal or observational evaluation of functional capacity; and adequate follow-up) (Clemson 2008).

We did not include other vision-correction interventions (e.g. cataract surgery, corrective lenses or filters) in this review.

\section{Types of outcome measures}

\section{Primary outcomes}

To be eligible for inclusion, the studies need to measure physical activity or falls. The primary outcomes were analyzed at 12 months of follow-up.

\section{A measure of physical activity.}

- Continuous objective measures, e.g. body fixed sensor activity monitoring.

- Continuous self-reported measures, e.g. validated questionnaires such as Physical Activity Scale for the Elderly, Community Healthy Activities Model Program for Seniors.

- Other self-reported measures, which may be dichotomous, e.g. single questions on physical activity.

A measure of falls.

- Falls (number of fallers or rate of falls) and injurious falls. Prospective daily calendars returned monthly for at least one year is the preferred method for recording falls (Lamb 2005).

\section{Secondary outcomes}

Our secondary outcomes, also analyzed at 12 months of follow-up, included the following.

- Fear of Falling, e.g. Short Falls Efficacy Scale-International (SFES-I) and the University of Illinois at Chicago Fear of Falling Measure.

- Quality of life, e.g. European Quality of Life (EuroQoL), 12-Item Short Form Health Survey (SF-12), 36-Item Short Form Health Survey.

Environmental and behavioural interventions for reducing physical activity limitation and preventing falls in older people with visual 
- Attitudes, beliefs and behaviors, e.g. Attitudes to Falls-Related Interventions Scale, Fear-Avoidance Beliefs Questionnaire. The latter may also be ascertained via qualitative methods such as focus groups and interviews.

\section{Follow-up}

As the frequency and duration of environmental and behavioral interventions varied depending on feasibility and severity of visual impairment, we used the time points (longer than or equal to two months) for outcome assessment as reported in each included trials in addition to 12 months.

\section{Search methods for identification of studies}

\section{Electronic searches}

The Cochrane Eyes and Vision Information Specialist searched the following electronic databases for randomized controlled trials and controlled clinical trials. There were no restrictions to language or year of publication. The electronic databases were last searched on 4 February 2020.

- Cochrane Central Register of Controlled Trials (CENTRAL; 2020, Issue 2) (which contains the Cochrane Eyes and Vision Trials Register) in the Cochrane Library (searched 4 February 2020) (Appendix 1).

- MEDLINE Ovid (1946 to 4 February 2020) (Appendix 2).

- Embase Ovid (1980 to 4 February 2020) (Appendix 3).

- Cumulative Index to Nursing and Allied Health Literature (CINAHL) EBSCO (1937 to 4 February 2020) (Appendix 4).

- Allied and Complementary Medicine Database (AMED) (January 1985 to 4 February 2020) (Appendix 5).

- OT Seeker (inception to 4 February 2020) (Appendix 6).

- metaRegister of Controlled Trials (mRCT) (www.controlledtrials.com; searched 4 February 2020) (Appendix 7).

- International Standard Research Clinical Trial Number (ISRCTN) registry (www.isrctn.com/editAdvancedSearch; searched 4 February 2020) (Appendix 8).

- US National Institutes of Health Ongoing Trials Register ClinicalTrials.gov (www.clinicaltrials.gov; searched 4 February 2020) (Appendix 9)

- World Health Organization (WHO) International Clinical Trials Registry Platform (ICTRP) (www.who.int/ictrp; searched 4 February 2020) (Appendix 10).

\section{Searching other resources}

We contacted authors of any ongoing trials or abstracts found, and searched the reference lists of full papers reviewed, as identified in our electronic search.

\section{Data collection and analysis}

\section{Selection of studies}

Two review authors, working independently, screened all titles and abstracts. Two review authors assessed the full-text articles of the selected titles and abstracts for eligibility according to the 'Criteria for considering studies for this review' outlined above. We resolved disagreements by consensus. In one instance, for an abstract, we sought additional information from the author. However, the study did not meet the inclusion criteria.

\section{Data extraction and management}

When a study fulfilled the inclusion criteria, two review authors independently extracted data concerning methodological issues, characteristics of participants, interventions and outcome measures, using a standard Covidence extraction form (Covidence). The review authors were not masked to the study authors, institutions or journal of publication. Where available and appropriate, we have presented quantitative data for the outcomes listed in the inclusion criteria in the analyses. Where studies reported standard errors of the means (SEs), we obtained standard deviations (SDs) by multiplying SEs by the square-root of the sample size. We attempted to contact authors of studies where there was inadequate reporting of data, to enable clarification and where appropriate.

\section{Assessment of risk of bias in included studies}

Two review authors worked independently to assess risk of bias in included studies using the methodology described in Chapter 8 of the Cochrane Handbook for Systematic Reviews of Interventions (Higgins 2019). The studies were assessed on the following criteria: random sequence generation, allocation concealment, masking (blinding) of participants and personnel, masking of outcome assessment, incomplete outcome data, selective outcome reporting and other sources of bias. Due to the nature of interventions, it was not possible to mask participants or staff providing the intervention. It was, however, possible to mask outcome assessors for measurements. Review authors' assessments in each domain were 'high risk of bias,' 'low risk of bias,' or 'unclear risk of bias.'

\section{Measures of treatment effect}

In each trial, we calculated risk ratios (RR) with 95\% confidence intervals $(\mathrm{CI})$ for dichotomous outcomes, incidence rate ratios (IRR) with $95 \% \mathrm{Cl}$ for outcomes reported as incidence rates, and mean differences (MD) with $95 \% \mathrm{Cl}$ for continuous outcomes (reporting mean and SD). We planned to calculate standardized mean differences (SMDs) and $95 \% \mathrm{Cls}$ when combining results from studies using different ways of measuring the same concept.

\section{Unit of analysis issues}

We reported the level at which randomization occurred in the included studies, as described in Chapter 9 of the Cochrane Handbook for Systematic Reviews of Interventions (McKenzie 2019). When individual was randomized, the unit of analysis was at individual level. Possible variations in study designs included cluster-randomized trials and cross-over trials. When such trials were available, we planned to assess whether trials had properly accounted for the intracluster or intraperson correlation.

\section{Dealing with missing data}

We analyzed available-case data as reported by assuming missing completely at random, meaning the probability of missing outcome data was the same for all participants in the study. We did not impute any missing data. We described the potential effect of missing data upon conclusions drawn from this review.

\section{Assessment of heterogeneity}

We assessed clinical heterogeneity by examining characteristics of individual studies. We assessed methodological heterogeneity by comparing study design and risk of bias across the trials. 
We planned to test statistical heterogeneity using the value of $I^{2}$ when meta-analysis was feasible, i.e. a value greater than $50 \%$ might indicate substantial heterogeneity (Deeks 2019). In the presence of heterogeneity, depending on the number of studies and the direction of effect, we would have combined the results of comparable groups of trials using the random-effects model and would have considered the subgroup analyses described later.

\section{Assessment of reporting biases}

We would have used funnel plots to test small study effects that could have been due to reporting bias, when a sufficient number of trials (10 or more) were identified (Egger 1997).

\section{Data synthesis}

We did not combine study results due to substantial clinical or statistical heterogeneity, but provided the effect estimates and associated $95 \% \mathrm{Cls}$ for individual trials. Where appropriate, we would have pooled results of comparable groups of studies using a random-effects model, and calculated effect estimates with their $95 \% \mathrm{Cls}$.

\section{Subgroup analysis and investigation of heterogeneity}

The review authors considered the following hypotheses using subgroup analysis if sufficient data were available.:

1. Are interventions equally effective on differing severities of visual impairment?

2. Are interventions equally effective with fallers at baseline as they are with the general older population?

There were insufficient data to look at these questions.

\section{Sensitivity analysis}

We planned to undertake sensitivity analyses, where indicated, to investigate the effects of methodological quality. For example, if appropriate, we would have looked at the effect of excluding QRCTs, as they would be at higher risk of selection bias. As no Q-RCTs were identified for inclusion in this review and there were few trials eligible for each comparison, we were unable to undertake such analyses.

\section{Grading of evidence and summary of findings table}

We present major outcomes (including physical activity, falls, fear of falling, and quality of life) in the 'Summary of findings' tables. We used the GRADE approach (GRADEpro 2015) to grade the quality of evidence per outcome for two main comparisons: 1) Environmental interventions versus social/home visits; and 2) Behavioral interventions versus usual activity or social/home visits. In GRADE, certainty of evidence may be ranked as follows: Highcertainty evidence, moderate-certainty evidence, low-certainty evidence and very low-certainty evidence.

We documented reasons for grading down evidence certainty with footnote.

\section{Summary of findings and assessment of the certainty of the evidence}

\section{RE S U L T S}

\section{Description of studies}

\section{Results of the search}

In the original version of this review, the electronic searches yielded a total of 6014 references from electronic databases, screening citations from relevant references. After removing duplicates and irrelevant reports, the authors screened the remaining 780 published reports to identify potentially relevant studies, and obtained full-text copies of 30 studies. After reading the full-text records, review authors excluded all the studies, while finding five ongoing studies.

We updated the search on 4 February 2020 and identified 2171 new records (Figure 1). We removed 27 duplicates and screened 2144 titles and abstracts for eligibility, of which we obtained 64 for full-text screening. We excluded one ongoing study and 55 other reports, with reasons for exclusion (Characteristics of excluded studies). We included one trial that was previously awaiting classification (Waterman 2016), one trial (Campbell 2005) that only reported fall-related outcomes and was excluded in previous review, and four new trials (Adams 2018; Gleeson 2017; Jeter 2015; Kovacs 2012). We included eight reports of six trials for this updated review, as well as one ongoing trial (ACTRN12607000399493). 
Figure 1. Study flow diagram.

No studies included in data
synthesis in previous version
of the review (search as of
November 2012)

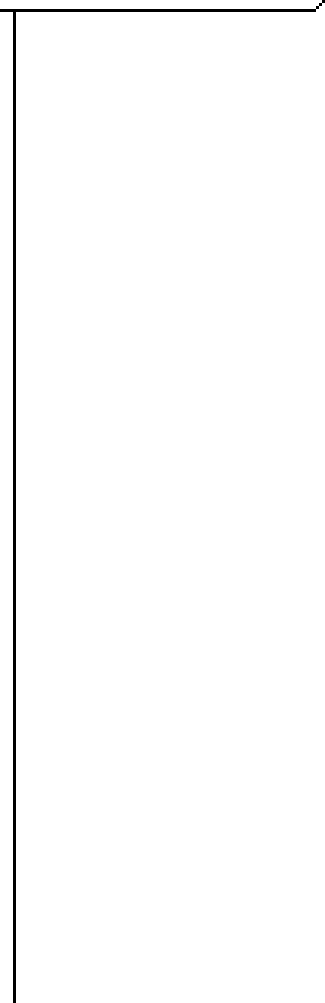

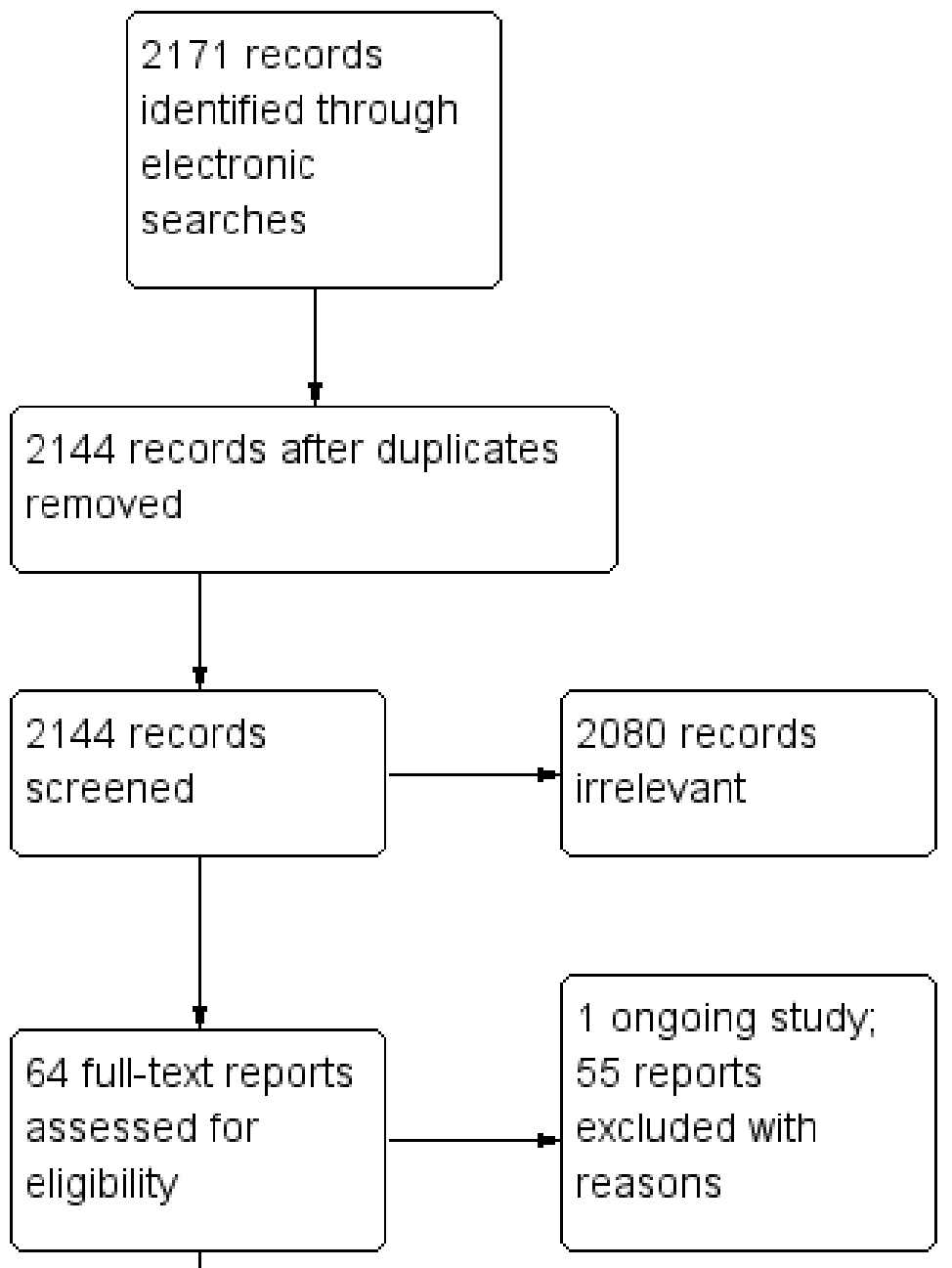

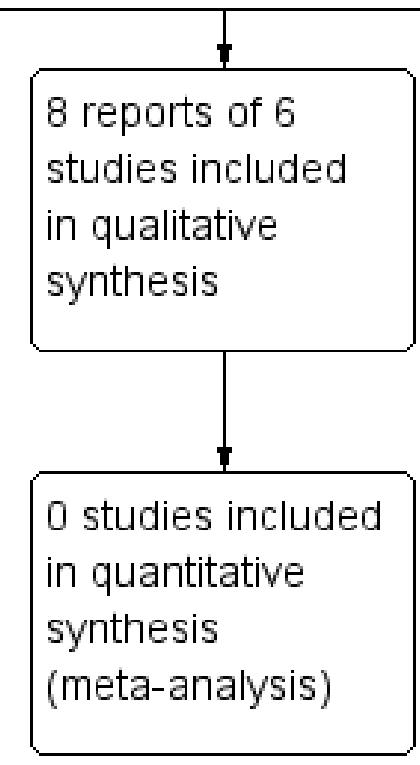

Environmental and behavioural interventions for reducing physical activity limitation and preventing falls in older people with visual 


\section{Included studies}

\section{Study design}

We describe the six included RCTs in Characteristics of included studies. Four trials randomized participants to one of two treatment groups, either an environmental or behavioral intervention, versus a control condition. One trial had three treatment groups (Waterman 2016) and another trial had four treatment groups (Campbell 2005), which included both environmental and behavioral interventions. The included trials were published between 2005 and 2018.

\section{Participants}

We included six RCTs (686 participants) conducted in five countries (Australia, Hungary, New Zealand, UK, US) with follow-up periods ranging from two to 12 months. Participants in these trials included older adults (mean age 80 years) and were mostly female (69\%), with visual impairments of varying severity and underlying causes. These trials varied in sample size, from 21 participants in the smallest trial (Jeter 2015) to 391 participants in the largest trial (Campbell 2005). Four trials included participants with varying severity and causes of visual impairment (Adams 2018; Campbell 2005; Kovacs 2012; Waterman 2016). Participants in one trial had age-related macular degeneration only (Gleeson 2017). Participants in another trial had blindness (Jeter 2015). Five trials included participants with an average age of 60 or older (Adams 2018; Campbell 2005; Gleeson 2017; Kovacs 2012; Waterman 2016). One trial included participants with a median age of 59 (Jeter 2015). We included this trial because half the participants were older than age 60. Five trials included both men and women (Adams 2018; Campbell 2005; Gleeson 2017; Kovacs 2012; Waterman 2016) and one included only women (Kovacs 2012). Overall, the followup periods ranged from eight weeks to 12 months. Five of six trials enrolled participants living independently in the community (Adams 2018; Campbell 2005; Gleeson 2017; Jeter 2015; Waterman 2016) and one trial recruited participants living in nursing homes (Kovacs 2012).

We observed clinical heterogeneity across six trials, including age, sex, reason and severity of visual impairments, and prior experience of environmental and behavioral training. Since older age at study enrolment, female sex, and poorer vision are associated with lower physical activity and higher risk of falls (Li 2017; Ong 2018; Ramulu 2019), the responses to environmental and behavioral interventions could vary by these factors.

\section{Interventions}

The included trials investigated a broad range of environmental and behavioral interventions. Two trials evaluated environmental interventions compared with social/home visits (Campbell 2005; Waterman 2016). Specifically, in Campbell 2005, occupational therapists visited the participants at home and assessed home safety using a checklist. They discussed potential hazards in the home that could lead to falls and implemented home modifications. In Waterman 2016, occupational therapists discussed environmental hazards present in their homes with participants, and jointly agreed upon an action plan about how to alter their environment to reduce risk of falls. The social/home visit was conducted by volunteers to provide social support and discuss general topics about lifestyles without giving any clinical advice. Five trials assessed the behavioral interventions versus usual activities or social/home visits (Adams 2018; Campbell 2005; Gleeson 2017; Jeter 2015; Kovacs 2012). These trials considered various behavioral interventions that were carried out by a greater range of healthcare professionals, including physiotherapists, occupational therapists and other exercise professionals. These behavioral interventions included the multi-component Falls Management Exercise program (Adams 2018), the Otago exercise program to improve muscle strength and balance (Campbell 2005; Kovacs 2012), the Alexander technique to improve movement and reaction to physical stimuli (Gleeson 2017), and Ashtangabased yoga therapy to alter stability and balance (Jeter 2015). Two trials evaluated the combination of home hazard modifications and Otago exercise program compared with social/home visits (Campbell 2005; Waterman 2016)

\section{Outcomes}

Two trials evaluated physical activity over six months follow-up (Adams 2018; Waterman 2016). Both trials reported a telephone questionnaire for self-reported physical activity at six months (Adams 2018; Waterman 2016), one trial used an instrumented monitoring of physical activity using a body fixed sensor at 6 months (Waterman 2016).

Four trials assessed a variety of measures of falls with study duration ranging from two to 12 months (Campbell 2005; Gleeson 2017; Kovacs 2012; Waterman 2016). Specifically, Kovacs 2012 reported number of fallers at two months and mean length of time to first fall. Gleeson 2017 reported number of fallers and fall rate per person at 12 months. Waterman 2016 reported number of fallers and injurious fallers at six months. Campbell 2005 reported number of fallers and injurious fallers at 12 months, falls per person year, injurious falls per person year, incidence rate ratio for falls and incidence rate ratio for injurious falls.

Four trials assessed fear of falling using different instruments with study durations ranging from two to six months (Adams 2018; Gleeson 2017; Jeter 2015; Waterman 2016). Specifically, Jeter 2015 used the University of Illinois at Chicago Fear of Falling instrument measured at two months. Three trials used the SFES-I instrument, one at three months (Gleeson 2017) and two at six months (Adams 2018; Waterman 2016).

Quality of Life measures, as one of the secondary outcomes, were also reported, including EuroQoL (Adams 2018), SF-12 (Waterman 2016), Geriatric Depression Scale (Gleeson 2017) and the Positive and Negative Affect Scale (Gleeson 2017). No adverse events due to the interventions were reported.

\section{Excluded studies}

Of 55 excluded studies in Characteristics of excluded studies, 21 (38\%) were non-randomized or quasi-randomized controlled trials, $17(31 \%)$ did not measure the outcomes of interest, 12 (22\%) did not report the comparison of interest, and five (9\%) did not study the population of interest.

\section{Risk of bias in included studies}

We evaluated the risk of bias for each trial using seven prespecified criteria, summarized in Figure 2. 
Figure 2. Risk of bias summary: review authors' judgements about each risk of bias item for each included study.

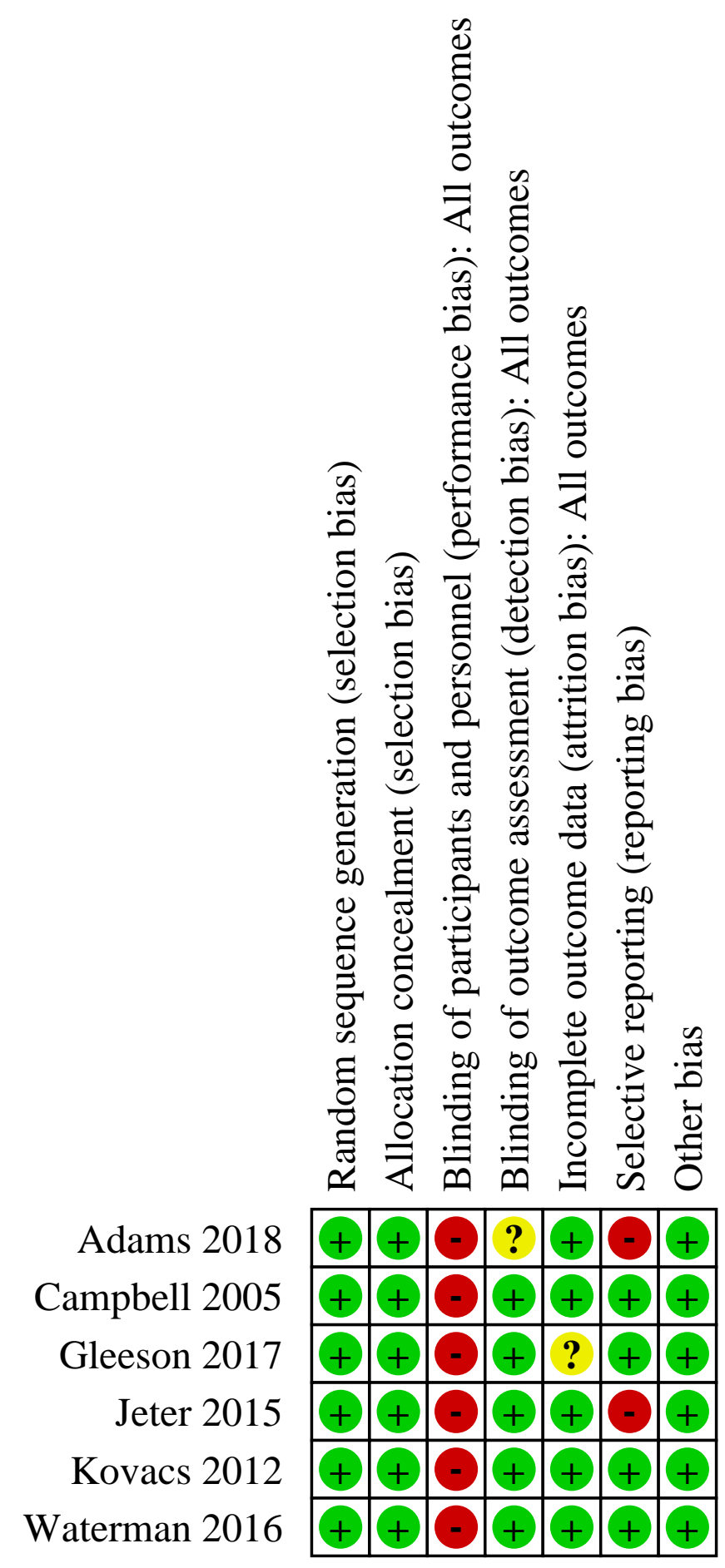

Environmental and behavioural interventions for reducing physical activity limitation and preventing falls in older people with visual 


\section{Allocation}

\section{Sequence generation}

We assessed six trials to be at low risk of bias for sequence generation (Adams 2018; Campbell 2005; Gleeson 2017; Jeter 2015; Kovacs 2012; Waterman 2016). All six trials used computer software to generate the random sequence.

\section{Allocation concealment}

We judged six trials to be at low risk of bias for allocation concealment. Five trials reported that the coordinators of group assignments did not know the next assignment when implementing the randomization (Adams 2018; Campbell 2005; Gleeson 2017; Jeter 2015; Waterman 2016). Kovacs 2012 used sealed, opaque envelopes.

\section{Blinding of participants and personnel (performance bias)}

It was not possible to mask participants or persons involved in the trial, due to the nature of the interventions. We judged all six trials to be at high risk of bias for blinding of participants and personnel (Adams 2018; Campbell 2005; Gleeson 2017; Jeter 2015; Kovacs 2012; Waterman 2016).

\section{Blinding of outcome assessment (detection bias)}

Five trials adequately described that outcome assessors remained masked to group allocation, and were judged to be at low risk of bias (Campbell 2005; Gleeson 2017; Jeter 2015; Kovacs 2012; Waterman 2016). Adams 2018 did not report whether the outcome assessor was masked, so we classified it as being at unclear risk of bias for this domain.

\section{Incomplete outcome data}

We provided the number of participants who were lost to followup or excluded after randomization in each trial (Characteristics of included studies). We considered five of six trials to be at low risk of bias because intention-to-treat analysis were adequately conducted, the number of dropouts was balanced between arms, or adequate reasons for dropouts were similar between arms (Adams 2018; Campbell 2005; Kovacs 2012; Waterman 2016; Jeter 2015). Gleeson 2017 reported that 10 of 120 participants did not complete assessments, so we classified it as being at unclear risk of bias for this domain.

\section{Selective reporting}

Four trials reported all prespecified outcomes in the trial registration or protocol, so we judged them to be at low risk of bias for this domain (Campbell 2005; Gleeson 2017; Kovacs 2012; Waterman 2016). Adams 2018 failed to show outcomes of activity avoidance and loneliness, both of which were reported in the trial registration. We assessed it to be at high risk of bias. Jeter 2015 reported results that were a subset of a larger battery of assessments that included psychological questionnaires and other information. We judged the risk of bias in this trial to be high for this domain.

\section{Other potential sources of bias}

We identified no other potential sources of bias in six trials.

\section{Effects of interventions}

See: Summary of findings 1 Summary of findings; Summary of findings 2 Summary of findings

The study characteristics varied across trials, including interventions and outcomes. As a result, we did not combine the quantitative results in meta-analysis. Instead, we reported the outcomes under each comparison when data were available.

We classified the six trials into six comparisons as described below. One three-arm trial (Waterman 2016) and one four-arm trial (Campbell 2005) contributed to more than one comparison.

- Environmental intervention versus social/home visits: two trials (Campbell 2005; Waterman 2016).

- Behavioral intervention versus social/home visits: five trials (Adams 2018; Campbell 2005; Gleeson 2017; Jeter 2015; Kovacs 2012)

- Environmental intervention versus behavioral: one trial (Campbell 2005)

- Environmental and behavioral intervention versus social/home visits: two trials (Campbell 2005; Waterman 2016).

- Environmental and behavioral intervention versus environmental: two trials (Campbell 2005; Waterman 2016).

- Environmental and behavioral intervention versus behavioral: one trial (Campbell 2005).

See: Summary of findings 1 and Summary of findings 2 for the main comparisons.

\section{Environmental intervention versus social/home visits (2 trials)}

\section{Physical activity and falls (2 trials, 224 participants)}

Two trials assessed home hazards modification compared with social/home visits (Campbell 2005; Waterman 2016 Figure 3) in older adults with visual impairment and living in the community. The follow-up period ranged from six to 12 months. 
Figure 3. Environmental intervention versus social/home visits comparison

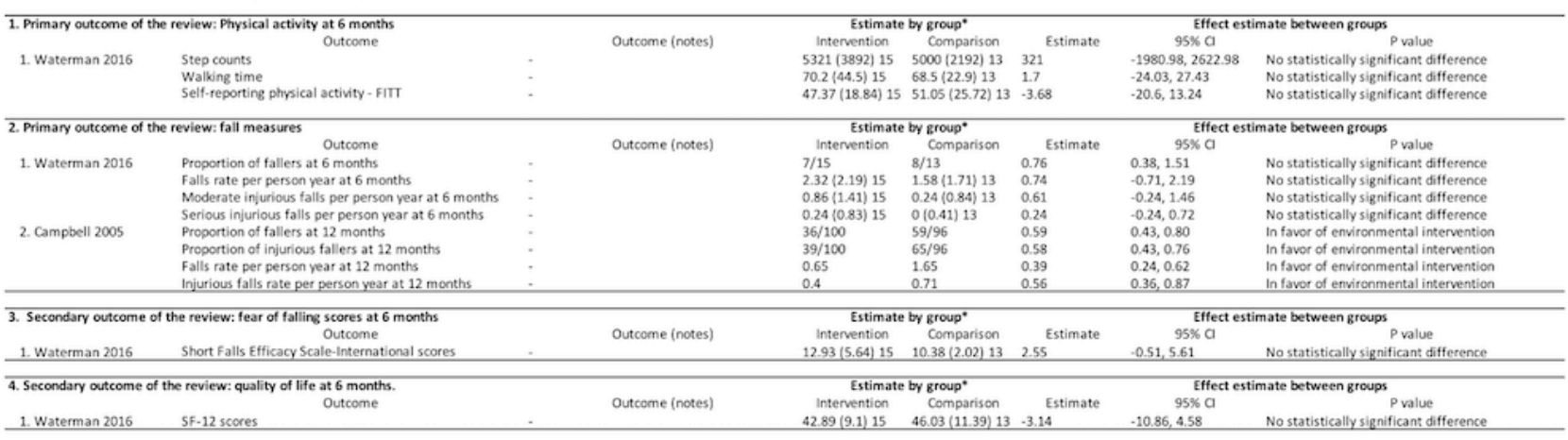

- Mean (standard deviation) sample size or Number of events/total
Gi: confidence interval

One trial assessed physical activity at six months (Figure 3) (Waterman 2016), Physical activity was measured using step counts, walking time, and self-reported activity (Phone-FITT). There were no significant difference in step counts (MD 321, 95\% $\mathrm{Cl}-1981$ to 2623 , walking time in minutes (MD 1.70, 95\% Cl -24.03 to 27.43 ) or self-reported activity (MD -3.68 scores, $95 \% \mathrm{Cl}-20.60$ to 13.24). The certainty of evidence was low, downgraded for risk of bias $(-1)$ and imprecision $(-1)$.

Two trials investigated a variety measures of falls ranging from six to 12 months. Waterman 2016 found no significant difference between groups at six months using the proportion of fallers (RR 0.76, 95\% $\mathrm{Cl} 0.38$ to 1.51 ), falls rate per person year (MD $0.74,95 \% \mathrm{Cl}-0.71$ to 2.19), moderate injurious falls per person year (MD 0.61, 95\% Cl -0.24 to 1.46 ), and serious injurious falls per person year (MD 0.24 $95 \% \mathrm{Cl}-0.24$ to 0.72 ). However, Campbell 2005 reported that home safety modification reduced falls (incidence rate ratio (IRR) 0.39 , $95 \% \mathrm{Cl} 0.24$ to 0.62 ) and injurious falls at 12 months (IRR $0.56,95 \%$ $\mathrm{Cl} 0.36$ to 0.87 ). The certainty of evidence was low, downgraded for risk of bias (-1) and imprecision (-1).

\section{Fear of falling and quality of life (one trial, 28 participants)}

In Waterman 2016, the mean score at six months was not significantly different between groups in SFES-I (MD 2.55 scores, $95 \% \mathrm{Cl}-0.51$ to 5.61 ) and SF-12 (MD -3.14 scores, $95 \% \mathrm{Cl}-10.86$ to 4.58). The certainty of evidence was low, downgraded for risk of bias $(-1)$ and imprecision (-1).

\section{Behavioral intervention versus social/home visits comparison (five trials)}

\section{Physical activity and falls (four trials, 415 participants)}

One trial assessed self-reported activity (Phone-FITT) at three months and six months (Figure 4) (Adams 2018). There was no evidence that physical activity differed between a Falls Management Exercise program and usual activity at three months (MD 8.00 scores, $95 \% \mathrm{Cl}-10.41$ to 26.41 ) and six months (MD 9.10 scores, $95 \% \mathrm{Cl}-13.85$ to 32.5$)$. The certainty of evidence was low, downgraded for risk of bias $(-1)$ and imprecision $(-1)$.

\section{Figure 4. Behavioural intervention versus usual activity or social/home visits comparison}

\begin{tabular}{|c|c|c|c|c|c|c|c|}
\hline \multicolumn{2}{|c|}{ 1. Primary outcome of the review: Physical activity at 6 months } & & \multicolumn{2}{|c|}{ Estimate by group*" } & \multicolumn{3}{|c|}{ Effect estimate between groups } \\
\hline 1. Adums 2018 & $\begin{array}{c}\text { Outcome } \\
\text { Self-reporting physical activity - FITT at } 3 \text { months }\end{array}$ & Outcome (notes) & $\begin{array}{l}\text { Intervention } \\
55(40.7) 29\end{array}$ & $\begin{array}{l}\text { Comparison } \\
47(131.1) 31\end{array}$ & Estimate & $\begin{array}{r}95 \% \mathrm{C} \\
-1044.2641\end{array}$ & $\begin{array}{l}\text { Pvalue } \\
\text { No statistically significant tfference }\end{array}$ \\
\hline & Self-reporting physical activity- FITT at 6 months & & $52.1(51.9) 28$ & $43(35.6) 31$ & 9.1 & $-13.85,32.5$ & No statistically signifieant difference \\
\hline \multirow{2}{*}{\multicolumn{2}{|c|}{ 2. Primary outcome of the review: fall measures }} & & \multicolumn{2}{|c|}{ Estimate by group" } & \multicolumn{3}{|c|}{ Effect estimate between groups } \\
\hline & & Outcome (notes) & Intervention & Comparison & Estimate & & Pvalue \\
\hline 1. Kovacs 2012 & Proportion of fallers at 6 months & & $8 / 21$ & $14 / 20$ & 0.54 & $0.29,1.01$ & No statistically significant difference \\
\hline & Mean length of time to first fall in weeks & & $18.5(7.36) 21$ & $14.8(8.32) 20$ & 3.70 & $-1.12,8.52$ & No statistically significant difference \\
\hline \multirow[t]{3}{*}{ 2. Campbell 2005} & Proportion of fallers at 12 months & & $47 / 97$ & $59 / 96$ & 0.79 & $0.61,1.02$ & No statistically significant difference \\
\hline & Proportion of injurious fallers at 12 months & & $53 / 97$ & $65 / 96$ & 0.89 & $0.70,1.13$ & No statistically significant difference \\
\hline & Falls rate per person year at 12 months & & 1.3 & 1.65 & 0.79 & $0.48,1.28$ & No statistically significant difference \\
\hline \multirow{4}{*}{ 3. Gleeson 2015} & $\begin{array}{l}\text { Injurious falls rate per person year at } 12 \text { months } \\
\text { Propoption of fallers at } 12 \text { moths }\end{array}$ & & $\begin{array}{l}0.57 \\
25 / 60\end{array}$ & 0.7170 & 0.82 & $0.48,1.40$ & 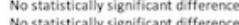 \\
\hline & $\begin{array}{l}\text { Proportion of fallers at } 12 \text { months } \\
\text { Proportion of injurious fallers at } 12 \text { months }\end{array}$ & : & $\begin{array}{l}25 / 60 \\
27 / 60\end{array}$ & $37 / 60$ & 0.73 & $\begin{array}{l}0.61,1.39 \\
0.52,1.03\end{array}$ & $\begin{array}{l}\text { No statistitacally yignificant difference } \\
\text { No statistically significant ofference }\end{array}$ \\
\hline & Falls rate per person year at 12 months & & 1.01 & 1.49 & 0.67 & $0.36,1.26$ & No statistically significant difference \\
\hline & Injurious falls rate per person year at 12 months & & & & 0.49 & $0.22,1.11$ & No statistically significant difference \\
\hline \multirow{2}{*}{\multicolumn{2}{|c|}{$\begin{array}{l}\text { 3. Secondary outcome of the review: fear of falling scores } \\
\text { Outcon }\end{array}$}} & & \multicolumn{2}{|c|}{ Estimate by grc } & \multicolumn{3}{|c|}{ Effect estimate between groups } \\
\hline & & Outcome (notes) & Intervention & Comparison & Estimate & & Pva \\
\hline \multirow{2}{*}{ 1. Adams 2018} & Short falls Efficacy Scale-Internation: & & $9(2.22) 29$ & $8(2.22) 30$ & 1.00 & $-0.13,2.13$ & gnificant difference \\
\hline & Short falls Efficacy Scale-Inter & & $8(2.96) 28$ & 8129 & 0.00 & $-1.51,1.51$ & gnificant difference \\
\hline \multirow{2}{*}{ 2. Gleeson 2017} & Short falls Efficacy Scale-International scores at 3 months & & $12.08(4.25) 55$ & $12.96(5.69) 59$ & .0 .88 & -2.72 .0 .96 & No statistically significant difference \\
\hline & Short falls Efficacy Scale-International scores at 12 & & $12.47(4.18) \mathrm{ss}$ & $12.7(5.65) 56$ & .0 .23 & $-2.08,1.62$ & No statistically significant difference \\
\hline 3. Jeter 2015 & illinois fear of falling scores at 2 months & Method of aggregation not reported & & & & & No statistically significant ofference \\
\hline \multicolumn{2}{|c|}{ 4. Secondary outcome of the review: quality of life at 6 months. } & & \multicolumn{2}{|c|}{ Estimate by group } & \multirow{2}{*}{\multicolumn{3}{|c|}{ Effect estimate between groups }} \\
\hline \multirow{3}{*}{ 1. Adıms 2018} & Outco & Outcome (notes) & Intervention & & Estimate & & \\
\hline & European quality of life- 15 scores at 3 months & & $.0 .20(0.22) 28$ & $-0.12(0.27) 31$ & .0 .08 & $-0.21,0.05$ & gnificart \\
\hline & European quality of life-15 scores at 6 months & & $-0.21(0.27) 28$ & $-0.05(0.28) 31$ & .0 .15 & $-0.29,-0.01$ & In favor of usual activity comparison \\
\hline \multirow[t]{3}{*}{ 2. Gleeson 2017} & Geriatric Depression Scale (GDS-5) at 3 months & & $1.29(1.39) 55$ & $1.34(1.47) 59$ & 0.15 & $-0.36,0.66$ & No statistically significant difference \\
\hline & Geriatric Depression Scale (GDS-S) at 12 months & & $1.13(1.16) 55$ & $1.14(1.37) 56$ & 0.01 & $-0.48,0.46$ & No statistically significant difference \\
\hline & $\begin{array}{l}\text { Positive and Negetivive Affect Scale (PANAS) at } 3 \text { months } \\
\text { Positive and Negaztive Affect Scale (PANAS) at } 12 \text { months }\end{array}$ & & $\begin{array}{l}36.04(6.695) 55 \\
35.04(8.55) 55\end{array}$ & $\begin{array}{l}3457(7.56) 59 \\
35.82(7.21) 56\end{array}$ & $\begin{array}{l}1.47 \\
.078\end{array}$ & $\begin{array}{r}-1.15,4.09 \\
-3.72 .216\end{array}$ & $\begin{array}{l}\text { No statisticially significiant difference } \\
\text { No statisticilly sisnoficant difference }\end{array}$ \\
\hline
\end{tabular}

- Mean (standard deviation) sample size or Number of events/total

Three trials evaluated the outcomes of falls at six months and/or 12 months using various measurement methods (Campbell 2005; Gleeson 2017; Kovacs 2012;). Kovacs 2012 reported no significant difference between the Otago exercise program in addition to a standard osteoporosis exercise program, compared with the standard osteoporosis exercise program alone, when evaluating

Environmental and behavioural interventions for reducing physical activity limitation and preventing falls in older people with visual 15 impairment (Review)

Copyright @ 2020 The Cochrane Collaboration. Published by John Wiley \& Sons, Ltd. 
the proportion of fallers at six months (RR $0.54,95 \% \mathrm{Cl} 0.29$ to 1.01 ) and mean length of time to first fall (MD $3.70,95 \% \mathrm{Cl}-1.12$ to 8.52 ). Campbell 2005 found no difference in falls (IRR $0.79,95 \% \mathrm{Cl} 0.48$ to 1.28 ) and injurious falls (IRR $0.82,95 \% \mathrm{Cl} 0.48$ to 1.40 ) at 12 months were found by comparing Otago exercise program plus vitamin $D$ supplementation with social visits. Likewise, in Gleeson 2017, no difference in falls (IRR $0.67,95 \% \mathrm{Cl} 0.36$ to 1.26 ) and injurious falls (IRR $0.49,95 \% \mathrm{Cl} 0.22$ to 1.11 ) at 12 months were reported by comparing Alexander technique with usual care. The certainty of evidence was low, downgraded for risk of bias (-1) and imprecision $(-1)$.

\section{Fear of falling and quality of life (three trials, 190 participants)}

Three trials assessed fear of falling scores at three to 12 months (Figure 4). Adams 2018 found the Falls Management Exercise program did not improve fear of falling compared with usual activity, using SFES-I scores at three months (MD 1.00 score, 95\% $\mathrm{Cl}-0.13$ to 2.13 ) and six months (MD $0,95 \% \mathrm{Cl}-1.51$ to 1.51 ). Gleeson 2017 reported that the Alexander technique did not change SFES-I scores compared with usual care at three months (MD -0.88 score, $95 \% \mathrm{Cl}-2.72$ to 0.96 ) and 12 months (MD -0.23 score, $95 \%$ $\mathrm{Cl}-2.08$ to 1.62). Likewise, in Jeter 2015, no significant difference was observed in the Illinois Fear of Falling score at two months by comparing Ashtanga-based yoga therapy and usual activity. Jeter 2015 did not report the mean or median (i.e. the method of aggregation). The certainty of evidence was low, downgraded for risk of bias (-1) and imprecision (-1).

One trial investigated European Quality of Life-15 at three and six months (Adams 2018). This trial reported that the Falls Management Exercise program improved quality of life at six months (MD -0.15 score, $95 \% \mathrm{Cl}-0.29$ to -0.01 ), but not at three months (MD -0.08 score, $95 \% \mathrm{Cl}-0.21$ to 0.05 ). The certainty of evidence was low, downgraded for risk of bias (-1) and imprecision $(-1)$.

One trial assessed Geriatric Depression Scale (GDS-5) and Positive and Negative Affect Scale (PANAS) at three and 12 months (Campbell 2005). No difference was noted by comparing the Otago exercise program with usual care for GDS-5 and PANAS.

\section{Environmental intervention versus behavioral comparison (one trial)}

\section{Falls (one trial, 197 participants)}

One trial compared home safety modification with the Otago exercise program on fall prevention at 12 months (Figure 5) (Campbell 2005). This trial found home safety modification reduced injurious falls (RR $0.66,95 \% \mathrm{Cl} 0.48$ to 0.91 ), but not all falls (RR 0.74 , $95 \% \mathrm{Cl} 0.53$ to 1.02 ).

\section{Figure 5. Environmental intervention versus behaviroural comparison}

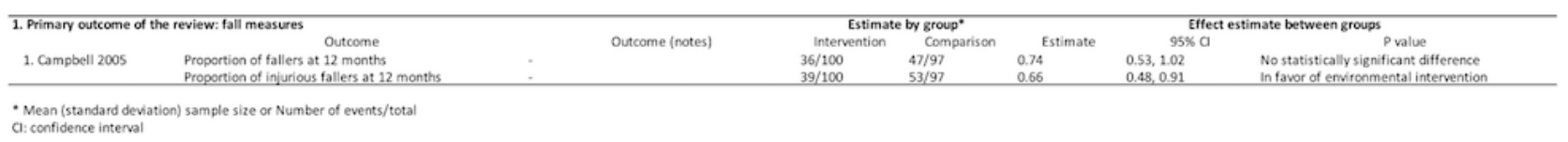

\section{Environmental and behavioral intervention versus social/ home visits comparison (two trials)}

\section{Physical activity and falls (two trials, 222 participants)}

One trial compared home hazards modification and the Otago exercise program with social/home visits on measurements of physical activity at six months (Figure 6) (Waterman 2016). There was no significant difference in step counts (MD -1073, 95\% Cl -2577 to 431 ), walking time in minutes (MD $-13.15,95 \% \mathrm{Cl}-31.18$ to 4.18 ) or self-reported activity (MD -5.36 scores, $95 \% \mathrm{Cl}-16.01$ to 5.29 ).

Figure 6. Environmental and behavioural intervention versus social/home visits comparison

\begin{tabular}{|c|c|c|c|c|c|c|c|}
\hline 1. Primary outcome of & eview: Physical activity at 6 months & & Estimat: & by group* & & Effect & \\
\hline & Outcome & Outcome (notes) & Intervention & Comparison & Estimate & $95 \times a$ & $p$ value \\
\hline 1. Waterman 2016 & Step counts & . & $3927(1815) 15$ & $5000(2192) 13$ & -1073 & $-2577.48,431.48$ & No statistically significant difference \\
\hline & $\begin{array}{l}\text { Walking time } \\
\text { Self-reporting phivical activity - FITT }\end{array}$ & & $\begin{array}{l}55(24.8) 15 \\
4456(1362) 15\end{array}$ & $68.5(22.9) 13$ & $\begin{array}{l}-13.15 \\
.536\end{array}$ & $-31.18,4.18$ & No statistically significant difference \\
\hline 2. Primary outcome of & 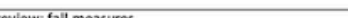 & & Fotime & $t_{0}$ & & Tlthene & 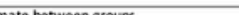 \\
\hline 2. Primary outcome of & eview: fall measures & Outcome (notes) & $\begin{array}{l}\text { Estimat } \\
\text { Intenention }\end{array}$ & by group" & Estimate & $\begin{array}{c}\text { Etfecte } \\
955 \times 9\end{array}$ & nate between groups \\
\hline 1. Waterman 2016 & Proportion of falless at 6 months & (S) & & $8 / 13$ & 097 & 054,177 & Ne statistically significant tifference \\
\hline & Falls rate per person year at 6 months & & $222(2.16) 15$ & $1.58(1.71) 13$ & 0.64 & 0.00208 & No statisticilly sispificant tifference \\
\hline & Moderate in iurious falls per person year at 6 months & & $0.37(0.99) 15$ & $0.24(0.84) 13$ & 0.13 & $-0.55,0.81$ & No statistically significant difference \\
\hline & Serious injurious falls per person year at 6 months & ${ }^{-}$ & $0(0.44) 15$ & $0(0.41) 13$ & 0.00 & .0 .32 .032 & No statistically significant difference \\
\hline 2. Campbell 2005 & Proportion of fallers at 12 months & & 47/98 & 59/96 & 0.78 & $0.60,1.01$ & No statistically significant difference \\
\hline & Proportion of injurious fallers at 12 months & & $61 / 98$ & $65 / 96$ & 1.01 & $0.81,1.26$ & No statistically significant difference \\
\hline & Falls rate per person year at 12 months & & 1.17 & 1.65 & & & \\
\hline & Injurious falls rate per person year at 12 months & & & 0.71 & & & \\
\hline 3. Secondary outcome & he review: fear of falling scores at 6 months & & Estimat: & by group" & & Effect e & nate between groups \\
\hline 1. & Outcome & Outcome (notes) & Intervention & Comparison & Estimate & $95 \times \mathrm{a}$ & Pvalue \\
\hline 1. Waterman 2016 & Short Falls Efficacy Scale-International scores & & $11.5(4.7) 15$ & $10.38(2.02) 13$ & 1.12 & $-1.50,3.74$ & No statistically significant difference \\
\hline 4. Secondary outcome & C review: quality of life at 6 months. & & Estimat: & by group* & & Effect e & mate between groups \\
\hline & Outcome & Outcome (notes) & Intervention & Comparison & Estimate & $95 \%$ a & pvalue \\
\hline 1. Waterman 2016 & SF-12 scores & & $43.21(8.61) 15$ & $46.03(11.39) 13$ & .2 .82 & $-10,39,4.75$ & No statistically significant difference \\
\hline
\end{tabular}

Two trials assessed different measures of falls at six and 12 months. Waterman 2016 found no significant difference between groups at six months using proportion of fallers (RR $0.97,95 \% \mathrm{Cl} 0.54$ to 1.77 ), falls rate per person year (MD $0.64,95 \% \mathrm{Cl}-0.80$ to 2.08 ), moderate injurious falls per person year (MD $0.13,95 \% \mathrm{Cl}-0.55$ to 0.81 ), and serious injurious falls per person year (MD $0,95 \% \mathrm{Cl}-0.32$ to 0.32 ). Similarly, Campbell 2005 reported the home safety modification did not reduce falls ( $\mathrm{RR} 0.78,95 \% \mathrm{Cl} 0.60$ to 1.01 ) and injurious falls 
at 12 months (RR $1.01,95 \% \mathrm{Cl} 0.81$ to 1.26 ), compared with the Otago exercise program.

\section{Fear of falling and quality of life (one trial, 28 participants)}

In Waterman 2016, there was no significant difference between groups in mean score at 6 months using SFES-I (MD 1.12 scores, 95\% $\mathrm{Cl}-1.05$ to 3.74 ) and SF-12 (MD -2.82 scores, $95 \% \mathrm{Cl}-10.39$ to 4.75 ) (Figure 6).

\section{Environmental and behavioral intervention versus environmental comparison ( 2 trials)}

\section{Physical activity and falls (2 trials, 228 participants)}

Waterman 2016 compared home hazards modification and the Otago exercise program with home hazard modification on measurements of physical activity at six months (Figure 7). There were no significant differences in step counts (MD $-1394,95 \% \mathrm{Cl}$ -3557 to 779 ), walking time in minutes (MD $-15.20,95 \% \mathrm{Cl}-40.98$ to 10.58 ) or self-reported activity (MD 4.60 scores, $95 \% \mathrm{Cl}-10.10$ to 19.30).

Figure 7. Environmental and behavioural intervention versus environmental comparison

\begin{tabular}{|c|c|c|c|c|c|c|c|}
\hline 1. Primary outcome of & review: Physical activity at 6 months & & Estimat: & by group" & & Effect & mate between groups \\
\hline & Outcome & Outcome (notes) & Intervention & comparison & Estimate & $95 \times a$ & Pvalue \\
\hline 1. Waterman 2016 & Step counts & & $3927(1815) 15$ & $5321(3892) 15$ & .1394 & $-3567.23,779.23$ & No statistically significant difference \\
\hline & Walking time & & $55(24.8) 15$ & $70.2(44.5) 15$ & -15.2 & $-40.98,10.58$ & No statistically significant difference \\
\hline & Self-reporting physical activity - FIIT & & $51.97(22.27) 15$ & $47.37(18.64) 15$ & 4.6 & $.10 .10,19.30$ & No statistically significant difference \\
\hline 2. Primary outcome o & review: fall measures & & Estimat: & by group" & & Effecte & mate between groups \\
\hline & Outcome & Outcome (notes) & Intervention & Comparison & Estimate & $95 \%$ a & \\
\hline 1. Waterman 2016 & Proportion of fallers at 6 months & & $9 / 15$ & $7 / 15$ & 0.78 & $0.39,1.54$ & No statistically significant difference \\
\hline & Falls rate per person year at 6 months & & $2.22(2.16) 15$ & $2.32(2.19) 15$ & -0.1 & $-1.66,1.46$ & No statistically significant difference \\
\hline & Moderate injurious falls per person year at 6 months & & $0.37(0.99) 15$ & $0.85(1.41) 15$ & -0.48 & $-1.35,0.39$ & No statistically significant difference \\
\hline & Serious injurious falls per person year at 6 months & & $0(0.44) 15$ & $0.24(0.83) 15$ & -0.24 & $-0.72,0.24$ & No statistically significant difference \\
\hline 2. Campbell 2005 & Proportion of fallers at 12 months & & $47 / 98$ & $36 / 100$ & 1.33 & $0.95,1.85$ & No statistically significant difference \\
\hline & Proportion of injurious fallers at 12 months & & $61 / 98$ & $39 / 100$ & & $1.17,2.09$ & In favor of environmental comparison \\
\hline & $\begin{array}{l}\text { Falls rate per person year at } 12 \text { months } \\
\text { Injurious falls rate per person year at } 12 \text { months }\end{array}$ & & $\begin{array}{l}1.17 \\
0.66\end{array}$ & $\begin{array}{l}0.65 \\
0.40\end{array}$ & & & . \\
\hline 3. Secondary outcom & he review: fear of falling scores at 6 months & & Estimat: & by group" & & Effect e & mate between groups \\
\hline 1. Waterman 2016 & Outcome & Outcome (notes) & Intervention & Comparison & Estimate & $95 \%$ a & Pvalue \\
\hline & & & $11.5(4.7) 15$ & $12.93(5.64)$ is & -1.43 & $-5.15,2.29$ & No statistically significant difference \\
\hline 4. Secondary outcome & e review: quality of life at 6 months. & & Estimat: & by group" & & Effect 5 & mate between groups \\
\hline 1. Waterman 2016 & SF-12 scores & Outcome (notes) & $\begin{array}{l}\text { Intervention } \\
43.218 .61 .15\end{array}$ & $\begin{array}{l}\text { Comparision } \\
4289(1.10) 15\end{array}$ & $\begin{array}{l}\text { Estimate } \\
0.32\end{array}$ & $\begin{array}{r}95 \% \mathrm{c} \\
-6.02,666\end{array}$ & $\begin{array}{l}\text { P value } \\
\text { Nostatistitallysignificant }\end{array}$ \\
\hline
\end{tabular}

Two trials evaluated measures of falls at six and 12 months. Waterman 2016 found no significant difference between groups at six months using proportion of fallers (RR $0.78,95 \% \mathrm{Cl} 0.39$ to 1.54 ), falls rate per person year (MD $-0.10,95 \% \mathrm{Cl}-1.66$ to 1.46 ), moderate injurious falls per person year (MD $-0.48,95 \% \mathrm{Cl}-1.35$ to 0.39 ), and serious injurious falls per person year (MD $-0.24,95 \% \mathrm{Cl}$ -0.72 to 0.24 ). Likewise, Campbell 2005 reported the home safety modification with the Otago exercise program reduced injurious falls (RR $1.56,95 \% \mathrm{Cl} 1.17$ to 2.09 ), but not all falls at 12 months (RR $1.33,95 \% \mathrm{Cl} 0.95$ to 1.85 ).

\section{Fear of falling and quality of life (one trial, 30 participants)}

In Waterman 2016, there was no significant difference between groups in mean score at six months using SFES-I (MD -1.43 scores,
$95 \% \mathrm{Cl}-5.15$ to 2.29 ) and SF-12 (MD 0.32 score, $95 \% \mathrm{Cl}-6.02$ to 6.66 ) (Figure 7).

\section{Environmental and behavioral intervention versus behavioral comparison (one trial)}

\section{Falls (one trial, 195 participants)}

One trial compared home safety modification and the Otago exercise program with the Otago exercise program alone on measures of falls at 12 months (Figure 8) (Campbell 2005). This trial found no significant difference between groups using proportion of fallers (RR $0.99,95 \% \mathrm{Cl} 0.74$ to 1.32 ) and proportion of injurious fallers (RR $1.14,95 \% \mathrm{Cl} 0.90$ to 1.45 ).

\section{Figure 8. Environmental and behavioural intervention versus behavioural comparison}

\begin{tabular}{|c|c|c|c|c|c|c|c|c|}
\hline \multirow{2}{*}{\multicolumn{3}{|c|}{ 1. Primary outcome of the review: fall measures }} & \multirow{4}{*}{ Outcome (notes) } & \multicolumn{2}{|c|}{ Estimate by group" } & \multicolumn{3}{|c|}{ Effect estimate between groups } \\
\hline \multirow{4}{*}{ 1. Campbell 2005} & & & & & Comparison & Estimate & & Pvalue \\
\hline & Proportion of fallers at 12 months & & & $47 / 98$ & 47/97 & 0.99 & $0.74,1.32$ & No statistically significant difference \\
\hline & Proportion of injurious fallers at 12 months & & & $61 / 98$ & $53 / 97$ & 1.14 & $0.90,1.45$ & No statistically significant difference \\
\hline & Falls rate per person year at 12 months & - & & 1.17 & & - & - & $\cdot$ \\
\hline
\end{tabular}

- Mean (standard dewation) sample size or Number of events/tota:

\section{DISCUSSION}

\section{Summary of main results}

This review included six RCTs of behavioral or environmental interventions in older people with vision impairment. We used both physical activity and falls as primary outcomes, because both measures are important physical domains in informing clinicians, patients, and other policy makers to make informed decisions.
The interpretation of the results for this review is complicated by the fact that environmental or behavioral interventions are not standard treatments, are often tailored individually and vary by severity of vision loss. Trial investigators reported physical activity and falls in many different ways, so we could not conduct metaanalysis.

There is no evidence of effect for most of the environmental or behavioral interventions studied for reducing physical activity limitation and preventing falls in visually impaired older people. 
We found low-certainty of evidence that the environmental interventions, particularly home safety modification delivered by occupational therapists, may provide a small benefit on preventing falls compared with social/home visits. These interventions did not appear to affect physical activity. No evidence of benefit was found with diverse types of behavioral interventions for reducing activity limitation and preventing falls, and the certainty of evidence was generally low.

\section{Overall completeness and applicability of evidence}

The six RCTs in this review included 686 older people (mean age $80)$, mostly women (69\%), with visual impairment. Participants characteristics varied due to enrolment method and inclusion criteria. Some trials generally recruited slightly younger people (median age 59) or those with less severe vision loss. In other trials, participants were more representative of older people with vision impairment, living in the community. Some trials recruited people through low vision clinics, or with specific eye diseases such as age-related macular degeneration. Some trials excluded participants with neurological disorders or who were unable to walk in their own residence, so that the results may not be applicable to older people with cognitive impairment or living in dependency. The included trials were conducted in five countries with different health care systems; therefore, the effectiveness of interventions could be sensitive to a variety of healthcare structures and networks settings. The results of this review should be interpreted with caution because four trials had low power to detect effect due to small sample size (Adams 2018; Jeter 2015; Kovacs 2012; Waterman 2016).

This review identified a variety of interventions for behavioral treatment, which precluded exploring the broad effect of different components as a whole. For example, differences between the Fall Management Exercise program and the Otago program. Also, usual care in our included studies was "no intervention but retaining access to normal health and vision care." Standardization of comparator groups would make it easier to consider studies for future meta-analyses.

We sought to identify fall-related measures, including proportion of fallers, proportion of injurious falls, fall rate per person year, injurious fall rate per person year, and mean length of time to first fall. Fall measures using both number of fallers and number of times a faller falls have clinical implications, because interventions may not prevent an individual from becoming a faller, but might prolong the time free from falls. Given the complexity of environmental and behavioral interventions and relatively small size of the six trials in this review, we cannot establish the applicability of the heterogeneous evidence in different settings and we do not know whether any benefits exist.

\section{Quality of the evidence}

The overall certainty of evidence showing the effectiveness of environmental and behavioral intervention is low, due to methodological limitations. We graded down by two levels. We had several reasons for this judgement. We judged each trial to be at high risk of bias in at least one domain. Fall-related measures were inconsistent across trials. Comparisons had small sample sizes. Few events in trials resulted in wide $95 \% \mathrm{Cls}$, crossing the line of no effect.
A major problem with the current evidence is that few of these trials measured widely agreed-upon outcomes with long followup periods (12 months or more) (Lamb 2005). Other limitations of the evidence were reflected in the clinical heterogeneity of the six trials, including demographic characteristics of participants (e.g. age, sex, and country of residence) and clinical characteristics (e.g. causes and severity of vision impairment and other comorbidities). Such clinical heterogeneity made it difficult to combine the effect estimates from individual trials in meta-analyses, to evaluate the overall effect.

\section{Potential biases in the review process}

We attempted to minimize bias by having two review authors independently review the titles and abstracts. We deliberately did not narrow our population and included both community-dwelling and care home/residential older adults, We believe we identified and included all evidence relevant to the population group of interest. It is possible that we may have been too precise in our inclusion criteria by excluding, for example, computerized visual field or eccentric view training. However, the focus of this review was to consider modifications to the home environment, and coping and enabling strategies to navigate safely in and out of the home. It was also difficult to identify studies that measured but did not report the outcomes (i.e. physical activity or falls). However, this is unlikely to have biased our study as these outcomes were not reported, and we provide the reason for excluding such studies as "outcome of interest not measured."

\section{Agreements and disagreements with other studies or reviews}

A Cochrane Review (Virgili 2010) considered the effectiveness of orientation and mobility training in adults with low vision. The review included two small trials comparing Orientation and Mobility training to physical exercise with weak evidence. Orientation and Mobility training had no effect in one study while it was found to be beneficial in the second. There is therefore little evidence on which type of Orientation and Mobility training is better for people with low vision who have specific characteristics and needs. This review is not specific to older adults with visual impairment.

Work commissioned by the Thomas Pocklington Trust (Ballinger 2009) aimed to carry out an evidence synthesis of qualitative research exploring the views and experiences of older people with visual impairment on participation in falls prevention initiatives. However, they found no studies that explicitly sought to explore the views of older visually impaired people. They included three studies with other relevant data. The single theme extending across all three studies was the capacity and desire for autonomous decision-making around environmental modification by older people with visual impairment, informed by but not dependent on the recommendations of others, such as health professionals. Other themes elicited included: the influence of function, ambience, safety, cleanliness and use of cues in decisionmaking about environmental modification. This research (Ballinger 2009) drew summaries based on the original version of this review (Skelton 2013).

Although there are previous systematic reviews of the effect of interventions to reduce falls in older adults (Cameron 2018; Hopewell 2018, Sherrington 2019), there is no specific review of 
those with visual impairment. However, one Cochrane Review (Gillespie 2012) did show that home safety interventions were more effective in reducing rate of falls and risk of falls in the higher risk subgroup of older people. They also found that there was some evidence that occupational therapy-led interventions on home safety assessment were more effective than non-occupational therapy-led interventions for reducing rate of falls, but this review was not performed on visually impaired people. Additionally, a recent review assessed the effectiveness of low vision rehabilitation on health-related quality of life and vision-related quality of life in visually impaired people (van Nispen 2020), but this review was not conducted on older adults (aged 60 or older). In van Nispen 2020, no evidence of benefit was reported for various types of low vision rehabilitation interventions on health-related quality of life, although the authors found low and moderate-certainty evidence of benefit by comparing psychological therapies versus usual care for vision-related quality of life. Most of the included studies in van Nispen 2020 on low vision rehabilitation had a short follow-up (six months or less).

A systematic review (Binns 2012) reminds researchers that outcome measures chosen to determine the effectiveness of low vision services should reflect capacity within daily activities, within the home environment, rather than just on clinical outcomes. This review only found seven trials, and whilst they felt able to confirm that rehabilitation services resulted in improved clinical and functional outcomes, they commented that the number of studies meeting their inclusion criteria was 'pitifully small.' Indeed, there is growing interest in physical activity in those living with low vision, as reflected by the advent of wearable technology which has created unprecedented opportunity to monitor real-world activity objectively that is often overlooked by questionnaires (Holbrook 2011; Schrack 2016). Future trials investigating whether interventions can make a difference to habitual activity should consider both objective and subjective assessments of physical activity.

\section{AUTHORS' CONCLUSIONS}

\section{Implications for practice}

There is no evidence of effect for most of the environmental or behavioral interventions studied for reducing physical activity limitation and preventing falls in visually impaired older people. However, there are only six eligible studies addressing this issue and these studies cannot be combined in meaningful ways.

Although behavioral interventions delivered by occupational therapists may reduce the rate of falls, we are unable to conclude if this is due to reduced activity restriction (increased mobility) or reduced activity (lessening exposure to risk). There are also limited and inconclusive results arising from the evaluation of environmental and behavioral interventions aimed at improving quality of life (de Jong 2019). This is perhaps because these intervention studies have not focused on coping strategies to engage with leisure activities and have instead focused on essential activities of daily living.

As restricted activity can lead to declining mobility, to potential distress and anxiety, and to an increased risk of falls, healthcare professionals need to consider ways to facilitate people to increase physical activity and prevent falls in older people with visual impairment.

\section{Implications for research}

There is a gap in knowledge concerning the effectiveness of environmental and behavioral interventions in reducing activity restriction and preventing falls in older people with irreversible vision loss. Future research, such as the ongoing trial described in Zijlstra 2009, considering the effectiveness of orientation and mobility training on activity restriction, physical activity, falls, fear of falling and quality of life in older adults with low vision, is necessary before any conclusions can be reached. Moreover, the physical activity program delivered through 'telerehabilitation' that enables remote prescription and monitoring of exercise may be considered as a feasible and safe technology for visually impaired people in future studies (Hutchinson 2018).

Of final note is the concern that interventions are rarely described in detail (Hoffmann 2014), nor do they provide information on the most appropriate participant groups or types of visual impairment with which they are most effective. It is important to customize interventions to suit particular individuals and their needs and preferences. A 'black box' of multiple interventions makes it difficult to pull out the effectiveness of different parts of the interventions, e.g. a mix of environmental and behavioral interventions may make it difficult to disentangle which type of intervention is more appropriate for which participant group.

\section{ACKNOWLEDGEMENTS}

The review authors would like to acknowledge the Cochrane Eyes and Vision (CEV), in particular, Iris Gordon, for compiling the search strategy and searches. We thank the following peer reviewers for their comments: Pradeep Ramulu (Wilmer Eye Institute), Nicola Adams (Northumbria University), and Danial Sayyad (consumer). We thank Claire Ballinger, Fiona Neil and Shelagh Palmer for their contributions to the published protocol of this review (Skelton 2011).

Dr. Tianjing Li (PI for CEV US satellite) was funded by UG1 EY020522 from the National Institutes of Health.

The views expressed in this publication are those of the authors and not necessarily those of the NIHR, NHS, NIH or the Department of Health.

This 2020 review update was managed by CEV@US and was signed off for publication by Tianjing Li and Richard Wormald. 


\section{R E F E R E N C E S}

\section{References to studies included in this review}

\section{Adams 2018 \{published data only\}}

Adams N, Skelton D, Howel D, Bailey C, Lampitt R, Fouweather $\mathrm{T}$, et al. Feasibility of trial procedures for a randomised controlled trial of a community based group exercise intervention for falls prevention for visually impaired older people: the VIOLET study. BMC Geriatrics 2018;18(1):307. [DOI: http://dx.doi.org/10.1186/s12877-018-0998-6]

\section{Campbell 2005 \{published data only\}}

Campbell AJ, Robertson MC, La Grow SJ, Kerse NM, Sanderson GF, Jacobs RJ, et al. Randomised controlled trial of prevention of falls in people aged $>$ or $=75$ with severe visual impairment: the VIP trial. BMJ 2005;331(7520):817. [DOI 10.1136/bmj.38601.447731.55]

\section{Gleeson 2017 \{published data only\}}

Gleeson M, Sherrington C, Lo S, Auld R, Keay L. Impact of the Alexander technique on well-being: a randomised controlled trial involving older adults with visual impairment. Clinical and Experimental Optometry 2017;100(6):633-41. [DOI: 10.1111/ cxo.12517]

Gleeson M, Sherrington C, Lo S, Keay L. Can the Alexander technique improve balance and mobility in older adults with visual impairments? A randomized controlled trial. Clinical Rehabilitation 2015;29(3):244-60. [DOI: 10.1177/0269215514542636]

\section{Jeter 2015 \{published data only\}}

Jeter P, Haaz-Moonaz S, Bittner Ak, Dagnelie G. Ashtangabased yoga therapy increases the sensory contribution to postural stability in visually-impaired persons at risk for falls as measured by the wii balance board: a pilot randomized controlled trial. PLOS One 2015;10(6):e0129646. [DOI: 10.1371/ journal.pone.0129646]

Jeter P, Roach C, Corson M, Ryan C, Nkodo AF, Haaz-Moonaz S, et al. Yoga reduces fear of falling and negative psychosocial states in persons with visual impairment. Journal of Alternative and Complementary Medicine 2014;20(5):A57. [DOI: http:// dx.doi.org/10.1089/acm.2014.5149]

\section{Kovacs 2012 \{published data only\}}

Kovacs E, Toth K, Denes L, Valasek T, Hazafi K, Molnar G, et al. Effects of exercise programs on balance in older women with age-related visual problems: a pilot study. Archives of Gerontology and Geriatrics 2012;55(2):446-52. [DOI: https:// dx.doi.org/10.1016/j.archger.2012.01.009]

\section{Waterman 2016 \{published data only\}}

Waterman H, Ballinger C, Brundle C, Chastin S, Gage H, Harper R, et al. A feasibility study to prevent falls in older people who are sight impaired: the VIP2UK randomised controlled trial. Trials 2016;17(1):464. [DOI: 10.1186/s13063-016-1565-0]

\section{References to studies excluded from this review}

Balliet 1985 \{published data only\}

Balliet R, Blood KM , Bach-y-Rita P. Visual field rehabilitation in the cortically blind? Journal of Neurology, Neurosurgery, and Psychiatry 1985;48(11):1113-24.

\section{Cumming 2007 \{published data only\}}

Cumming RG, Ivers R, Clemson L, Cullen J, Hayes MF, Tanzer M, et al. Improving vision to prevent falls in frail older people: a randomised trial. Journal of the American Geriatrics Society 2007;55(2):175-81.

Dahlin-Ivanoff 2002 \{published data only\}

Dahlin Ivanoff S, Sonn U, Svensson E. A health education program for elderly persons with visual impairments and perceived security in the performance of daily occupations: a randomised study. American Journal of Occupational Therapy 2002;56(3):322-30.

\section{Day 2002 \{published data only\}}

Day L, Fildes B, Gordon I, Fitzharris M, Flamer H, Lord S. Randomised factorial trial of falls prevention among older people living in their own homes. BMJ 2002;325(7356):128.

\section{Deemer 2017 \{published data only\}}

Deemer A, Massof R, Rovner B, Casten R, Piersol C. Functional outcomes of the low vision depression prevention trial in agerelated macular degeneration. Investigative Ophthalmology \& Visual Science 2017;58(3):1514-1520. [DOI: 10.1167/ iovs.16-20001]

Rovner B, Casten R, Hegel M, Massof R, Leiby B, Ho A et al. Improving function in age-related macular degeneration: a randomized clinical trial. Ophthalmology 2013;120(8):1649-1655. [DOI: 10.1016/j.ophtha.2013.01.022]

Rovner B, Casten R, Hegel M, Massof R, Leiby B, Ho A, et al. Low vision depression prevention trial in age-related macular degeneration: a randomized clinical trial. Ophthalmology 2014;121(11):2204-2211. [DOI: 10.1016/j.ophtha.2014.05.002]

\section{de Hann 2016 \{published data only\}}

de Haan GA, Melis-Dankers BJ, Brouwer WH, Tucha O, Heutink J. The effects of compensatory scanning training on mobility in patients with homonymous visual field defects: further support, predictive variables and follow-up. PLOS One 2016;11(12):e0166310. [DOI: 10.1371/journal.pone.0166310]

de Haan GA, Melis-Dankers BJ, Brouwer WH, Tucha O, Heutink $J$. The effects of compensatory scanning training on mobility in patients with homonymous visual field defects: a randomized controlled trial. PLOS One 2015;10(8):e0134459. [DOI: 10.1371/ journal.pone.0134459]

\section{Deremeik 2007 \{published data only\}}

Deremeik J, Broman AT, Freidman D, West SK, Massof R, Park W, et al. Low vision rehabilitation in a nursing home population: The SEEING study. Journal of Visual Impairment and Blindness 2007;101(11):701-14. 
Dillon 2018 \{published data only\}

Dillon L, Clemson L, Coxon K, Keay L. Understanding the implementation and efficacy of a home-based strength and balance fall prevention intervention in people aged 50 years or over with vision impairment: a process evaluation protocol. BMC Health Servce Research 2018;18(1):512. [DOI: https:// dx.doi.org/10.1186/s12913-018-3304-6]

\section{Elliott 2016 \{published data only\}}

Elliott DB, Hotchkiss J, Scally AJ, Foster R, Buckley JG. Intermediate addition multifocals provide safe stair ambulation with adequate 'short-term' reading. Ophthalmic and Physiological Optics 2016;36(1):60-8. [DOI: http:// dx.doi.org/10.1111/opo.12236]

\section{Girdler 2010 \{published data only\}}

Girdler SJ, Boldy DP, Dhaliwal SS, Crowley M, Packer TL. Vision self-management for older adults: a randomised controlled trial. British Journal of Ophthalmology 2010;94(2):223-8.

\section{Gleeson 2014 \{published data only\}}

Gleeson M, Sherrington C, Keay L. Exercise and physical training improve physical function in older adults with visual impairments but their effect on falls is unclear: a systematic review. Journal of Physiotherapy 2014;60(3):130-5. [DOI: https:// dx.doi.org/10.1016/j.jphys.2014.06.010]

\section{Gutman 2002 \{published data only\}}

Gutman C, Jaffe K. Group intervention for visually impaired grandparents. Journal of Visual Impairment and Blindness 2002;96(10):741-3.

\section{Hollands 2013 \{published data only\}}

Hollands KL, Pelton T, Wimperis A, Whitham D, Jowett S, Sackley $C$, et al. Visual cue training to improve walking and turning after stroke: a study protocol for a multi-centre, single blind randomised pilot trial. Trials 2013;14:276. [DOI: https:// dx.doi.org/10.1186/1745-6215-14-276]

\section{Hollands 2015 \{published data only\}}

Hollands KL, Pelton TA, Wimperis A, Whitham D, Tan W, Jowett $S$, et al. Feasibility and preliminary efficacy of visual cue training to improve adaptability of walking after stroke: multi-centre, single-blind randomised control pilot trial. PLOS One 2015;10(10):e0139261. [DOI: https://dx.doi.org/10.1371/ journal.pone.0139261]

\section{Holloway 2018 \{published data only\}}

Holloway E, Constantinou M, Xie J, Fenwick EK, Finkelstein EA, Man RE, et al. Improving eye care in residential aged care facilities using the Residential Ocular Care (ROC) model: Study protocol for a multicentered, prospective, customized, and cluster randomized controlled trial in Australia. Trials 2018;19(1):650. [DOI: http://dx.doi.org/10.1186/ s13063-018-3025-5]

\section{Keay 2018 \{published data only\}}

Keay L, Dillon L, Clemson L, Tiedemann A, Sherrington C, McCluskey $P$, et al. PrevenTing Falls in a high-risk, visionimpaired population through specialist ORientation and Mobility services: protocol for the PlaTFORM randomised trial. Injury Prevention 2018;24(6):459-66. [DOI: http://

dx.doi.org/10.1136/injuryprev-2016-042301]

Kuyk 2008 \{published data only\}

Kuyk T, Liu L, Elliott JL, Grubbs HE, Owsley C, McGwim G Jr, et al. Health-related quality of life following blind rehabilitation. Quality of Life Research 2008;4(17):497-507.

\section{Kuyk 2010 \{published data only\}}

Kuyk T, Liu L, Elliott J, Fuhr B. Visual search training and obstacle avoidance in adults with visual impairments. Journal of Visual Impairment and Blindness 2010;104(4):215-27.

La Grow 2004 \{published data only\}

La Grow SJ. The effectiveness of comprehensive low vision services for older persons with visual impairments in New Zealand. Journal of Visual Impairment and Blindness 2004;98(11):679-92.

\section{Machado 2014 \{published data only\}}

Machado K, Domiciano DS, Machado LG, Figueiredo CP, Lopes JB, Caparbo V, et al. Frequency and risk factors for recurrent falls in community-dwelling elderly: A populationbased prospective cohort study in Brazil. The Sao Paulo ageing \& health (SPAH) study. Arthritis and Rheumatology 2014;10:S30-1. [DOI: http://dx.doi.org/10.1002/art.38914]

\section{McCabe 2000 \{published data only\}}

McCabe P, Nason F, Demers Turco P, Friedman D, Seddon JM. Evaluating the effectiveness of a vision rehabilitation intervention using an objective and subjective measure of functional performance. Ophthalmic Epidemiology 2000;7(4):259-70.

\section{NCT00545220 \{unpublished data only\}}

NCT00545220. Problem solving training and low vision rehabilitation. clinicaltrials.gov/ct2/show/NCT00545220 (first received 17 October 2007).

\section{NCT00769015 \{unpublished data only\}}

NCT00769015. Low vision depression prevention trial for age related macular degeneration (VITAL). clinicaltrials.gov/show/ NCT00769015 (first received 8 October 2008).

NCT04066075 \{published data only\}

NCT04066075. Beacon sensors and telerehabilitation for low vision. clinicaltrials.gov/ct2/show/NCT04066075 (first received 26 August 2019).

\section{NCT04084587 \{published data only\}}

NCT04084587. Visual rehabilitation through acoustic biofeedback with retimax vision trainer in patients with advanced age-related macular degeneration. clinicaltrials.gov/ ct2/show/NCT04084587 (first received 10 September 2019).

\section{Ni 2012 \{published data only\}}

Ni W, Li X, Ao M, Zhang H, Hou Z, Si S, et al. Using the reallife vision test to assess the functional vision of age-related cataract patients. Eye 2012;26(11):1402-11. [DOI: http:// dx.doi.org/10.1038/eye.2012.168]

Environmental and behavioural interventions for reducing physical activity limitation and preventing falls in older people with visual 
Overbury 1996 \{published data only\}

Overbury O, Quillman RD. Perceptual learning in adventitious low vision: task specificity and practice. Journal of Vision Rehabilitation 1996;10(1):7-14.

\section{Pankow 2004 \{published data only\}}

Pankow L, Luchins D, Studebaker J, Chettleburgh D. Evaluation of a vision rehabilitation program with older adults with visual impairment. Topics in Geriatric Rehabilitation 2004;20(3):223-32.

\section{Rees 2015 \{published data only\}}

Lamoureux E, Chiang PPC, Marella M, Hassell J, Larizza M, Keeffe J, et al. A randomized controlled trial of a selfmanagement program for low vision implemented in low vision rehabilitation services. Clinical and Experimental Ophthalmology 2012;1:125. [DOI: http://dx.doi.org/10.1111/ ceo.12011]

Rees G, Xie J, Chiang P, Larizza M, Marella M, Hassell J, et al. A randomised controlled trial of a self-management programme for low vision implemented in low vision rehabilitation services. Patient Education and Counseling 2015;98(2):174-181. [DOI: 10.1016/j.pec.2014.11.008]

\section{Reeves 2004 \{published data only\}}

Reeves BC, Harper RA, Russell WB. Enhanced low vision rehabilitation for people with age related macular degeneration: a randomised controlled trial. British Journal of Opthalmology 2004;88(11):1443-49.

\section{Renaud 2013 \{published data only\}}

Renaud J, Bedard E. Depression in the elderly with visual impairment and its association with quality of life. Clinical Interventions in Aging 2013;8:931-43. [DOI: https:// dx.doi.org/10.2147/CIA.S27717]

\section{Renieri 2013 \{published data only\}}

Renieri G, Pitz S, Pfeiffer N, Beutel ME, Zwerenz R. Changes in quality of life in visually impaired patients after low-vision rehabilitation. International Journal of Rehabilitation Research 2013;36(1):48-55.

\section{Riazi 2014 \{published data only\}}

Riazi A, Walters K, Rubin G, Ambler G, Jichi F, Mynors-Wallis L, et al. A pilot randomised controlled trial of Problem-Solving Treatment for Visual Impairment (POSITIVE): protocol paper. Ophthalmic and Physiological Optics 2014;34(4):489-97. [DOI: 10.1111/opo.12135]

\section{Ribeiro 2015 \{published data only\}}

Ribeiro MV, Hasten-Reiter Junior HN, Ribeiro EA, Juca MJ, Barbosa FT, Sousa-Rodrigues CF. Association between visual impairment and depression in the elderly: a systematic review. Arquivos Brasileiros De Oftalmologia 2015;78(3):197-201. [DOI: http://dx.doi.org/10.5935/0004-2749.20150051]

\section{Roets Merken 2015 \{published data only\}}

Roets-Merken LM, Draskovic I, Zuidema SU, van Erp WS, Graff MJ, Kempen GI, et al. Effectiveness of rehabilitation interventions in improving emotional and functional status in hearing or visually impaired older adults: a systematic review with meta-analyses. Clinical Rehabilitation 2015;29(2):107-19. [DOI: https://dx.doi.org/10.1177/0269215514542639]

Roqueta 2016 \{published data only\}

Roqueta C, Arnau-Barres I, Ronquillo N, Martin M, Vazquez O, Miralles R. Risk factors for falling, benzodiazepines and fear of falling: Is there any relationship? European Geriatric Medicine 2016;7(Suppl 1):S154.

\section{Rottman Sagebiel 2017 \{published data only\}}

Rottman-Sagebiel RA, Reilly M, Corbo J, Espinoza S. Fall prevention in older community dwelling patients. Journal of the American Geriatrics Society 2017;65(Suppl 1):S40. [DOI: http:// dx.doi.org/10.1111/\%28ISSN\%291445-5994]

Rubin 2017 \{published data only\}

Rubin G. First results from the EFFECT Trial, an RCT of eccentric viewing training for patients with AMD. Investigative Ophthalmology and Visual Science. 2017;58(8):ARVO E-abstract 4766.

\section{Rumrill 1999 \{published data only\}}

Rumrill PD. Effects of a social competence training program on accomodation request activity, situational self efficacy, and Americans with disabilities act knowledge among employed people with visual impairments and blindness. Journal of Vocational Rehabilitation 1999;12(1):25-31.

\section{Sand 2016 \{published data only\}}

Sand KM, Wilhelmsen G, Naess H, Midelfart A, Thomassen L, Hoff JM. Vision problems in Ischaemic stroke patients: Effects on life quality and disability. European Journal of Neurology 2016;23(Suppl 1):1-7. [DOI: http://dx.doi.org/10.1111/ ene.12848]

\section{Scanlan 2004 \{published data only\}}

Scanlan JM, Cuddeford JE. Low vision rehabilitation: A comparison of traditional and extended teaching programs. Journal of Visual Impairment and Blindness 2004;98(10):601-10.

Sekeryapan 2013 \{published data only\}

Sekeryapan B, Sabri Balik M, Oner V, Guvercin Y, Turkyilmaz K, Erkut $A$, et al. The relation between visual impairment and extremity fractures. Türk Oftalmoloji Dergisi 2013;43(4):250-2. [DOI: http://dx.doi.org/10.4274/tjo.43.29292]

Shi 2013 \{published data only\}

Shi J, Yu PL. Prospective study of falls and fall risk factors in community dwelling elderly people in Beijing. Journal of the American Geriatrics Society 2013;3:S359. [DOI: http:// dx.doi.org/10.1111/jgs.12439]

Shi 2016 \{published data only\}

Shi J, Yu PL, Yao HQ, Tao YK, Zhou BY, Duan CB, et al. Prospective study of falls and risk factors for falls in communitydwelling elderly people in Beijing. Journal of the American Geriatrics Society 2016;64(Suppl 2):S379.

Siantar 2015 \{published data only\}

Siantar RG, Cheng CY, Gemmy Cheung CM, Lamoureux EL, Ong PG, Chow KY, et al. Impact of visual impairment and eye

Environmental and behavioural interventions for reducing physical activity limitation and preventing falls in older people with visual 
diseases on mortality: the Singapore Malay Eye Study (SiMES). Scientific Reports 2015;5:16304. [DOI: http://dx.doi.org/10.1038/ srep16304]

\section{Skalska 2013 \{published data only\}}

Skalska A, Wizner B, Piotrowicz K, Klich-Raczka A, Klimek E, Mossakowska $\mathrm{M}$, et al. The prevalence of falls and their relation to visual and hearing impairments among a nation-wide cohort of older Poles. Experimental Gerontology 2013;48(2):140-6. [DOI: https://dx.doi.org/10.1016/j.exger.2012.12.003]

\section{Skelton 2016 \{published data only\}}

Skelton D, Bailey C, Howel D, Cattan M, Deary V, Coe D, et al. Visually impaired older people's exercise programme for falls prevention (VIOLET): a feasibility study protocol. BMJ Open 2016;6(8):e011996. [DOI: 10.1136/bmjopen-2016-011996]

\section{Smith 2009 \{published data only\}}

Smith TM, Thomas K, Dow K. The effect of an educational program for persons with macular degeneration; A pilot study. Journal of Visual Impairment and Blindness 2009;103(4):234-40.

\section{Smith 2017 \{published data only\}}

Smith AA, Silva AO, Rodrigues RA, Moreira MA, Nogueira JA, Tura LF. Assessment of risk of falls in elderly living at home. Revista Latino-Americana de Enfermagem 2017;25:e2754. [DOI: http://dx.doi.org/10.1590/1518-8345.0671.2754]

\section{Stelmack 2005 \{published data only\}}

Stelmack J, Mancil R, Mancil G, Cummings R, Moran D, Rinne S, et al. Veterans affairs low vision intervention trial (LOVIT). Investigative Ophthalmology and Visual Science 2005;46:ARVO EAbstract 1920.

Stelmack J, Tang C, Reda D, Rinne S, Mancil R, Cummings RW, et al. The veterans affairs low vision intervention trial follow up: twelve month outcomes. American Academy of Optometry 2008;12:644-7.

Stelmack JA, Rinne S, Mancil RM, Dean D, Moran D, Tang XC, et al. Successful outcomes from a structured curriculum used in the Veterans Affairs Low intervention Trial. Journal of Visual Impairment and Blindness 2008;102(10):636-48.

Stelmack JA, Tang XC, Reda DJ, Rinne S, Mancil RM, Massof RW, et al. Outcomes of the veteran affairs low vision intervention trial (LOVIT). Archives of Ophthalmology 2008;126(5):608-17.

\section{Sturrock 2015 \{published data only\}}

Sturrock BA, Xie J, Holloway EE, Lamoureux EL, Keeffe JE, Fenwick EK, et al. The influence of coping on vision-related quality of life in patients with low vision: A prospective longitudinal study. Investigative Ophthalmology and Visual Science 2015;56(4):2416-22. [DOI: http://dx.doi.org/10.1167/ iovs.14-16223]

\section{Vukicevic 2009 \{published data only\}}

Vukicevic M, Fitzmaurice K. Eccentric viewing training in the home environment: can it improve performance of activities of daily living? Journal of Visual Impairment and Blindness 2009;103(5):277-90.
Wang 2012 \{published data only\}

Wang MY, Rousseau J, Boisjoly H, Schmaltz H, Kergoat MJ, Moghadaszadeh S, et al. Activity limitation due to a fear of falling in older adults with eye disease. Investigative Ophthalmology and Visual Science 2012;53(13):7967-72. [DOI: https://dx.doi.org/10.1167/iovs.12-10701]

\section{West 2004 \{published data only\}}

West SK, Friedman DF, Broman AT, Munoz B, Bandeen-Roche K, Deremeik J, et al. Effect of a vision restoration/rehabilitation program on physical function in a population of nursing home residents with visual loss. SEEING Clinical Trial. Investigative Ophthalmology and Visual Science 2004;45:ARVO E-Abstract 1362.

\section{Zijlstra 2009 \{unpublished data only\}}

Zijlstra GA, van Rens GH, Scherder EJ, Brouwer DM, van der Velde J, Verstraten BF, et al. Effects and feasibility of a standardised orientation and mobility training in using an identification cane for older adults with low vision: design of a randomised controlled trial. BMC Health Services Research 2009;9:153.

\section{References to ongoing studies}

\section{ACTRN12607000399493 \{unpublished data only\}}

ACTRN12607000399493. A randomised controlled trial of a low vision self management program on quality of life in people with low vision. apps.who.int/trialsearch/Trial.aspx? TrialID=ACTRN12607000399493 (first received 13 December 2012).

\section{Additional references}

\section{Ballinger 2009}

Ballinger C, Neil F, Howe T, Skelton DA, Palmer S, Gray L. A qualitative evidence synthesis of the views and experiences of older people with visual impairment about interventions to reduce falls. Thomas Pocklington Trust 2009.

\section{Berger 2012}

Berger S. Is my world getting smaller? The challenges of living with vision loss. Journal of Visual Impairment and Blindness 2012;106(1):5-16.

\section{Binns 2012}

Binns A, Bunce C, Dickinson C, Harper R, Tudor-Edwards R, Woodhouse $\mathrm{M}$, et al. How effective is low vision service provision? A systematic review. Survey of Opthalmology 2012;57(1):34-65.

\section{Brouwer 2008}

Brouwer D, Sadlo G. Limitations in mobility: experiences of visually impaired older people. British Journal of Occupational Therapy 2008;71(10):414-21.

\section{Cameron 2018}

Cameron ID, Dyer SM, Panagoda CE, Murray GR, Hill KD, Cumming RG, et al. Interventions for preventing falls in older people in care facilities and hospitals. Cochrane Database of

Environmental and behavioural interventions for reducing physical activity limitation and preventing falls in older people with visual 
Systematic Reviews 2018, Issue 9. Art. No: CD005465. [DOI: 10.1002/14651858.CD005465.pub4]

\section{Clemson 2003}

Clemson L, Manor D, Fitzgerald MH. Behavioral factors contributing to older adults falling in public places. OTJR: Occupation, Participation and Health 2003;23(3):107-17.

\section{Clemson 2008}

Clemson L, Mackenzie L, Ballinger C, Close JC, Cumming RG. Environmental interventions to prevent falls in communitydwelling older people: a meta-analysis of randomized trials. Journal of Aging Health 2008;20(8):954-71.

\section{Covidence [Computer program]}

Veritas Health Innovation Covidence. Melbourne, Australia: Veritas Health Innovation, accessed 28 February 2020. Available at www.covidence.org.

\section{Crews 2004}

Crews JE, Campbell VA. Vision impairment and hearing loss among community-dwelling older Americans: implications for health and functioning. American Journal of Public Health 2004;94(5):823-9.

\section{de Jong 2019}

de Jong LD, Coe D, Bailey C, Adams N, Skelton DA. Views and experiences of visually impaired older people and exercise instructors about the Falls Management Exercise programme: a qualitative study. Disability and Rehabilitation 2019;41:1-7. [DOI: 10.1080/09638288.2019.1704894]

\section{Deeks 2019}

Deeks JJ, Higgins JPT, Altman DG. Chapter 10: Analysing data and undertaking meta-analyses. In: Higgins JPT, Thomas J, Chandler J, Cumpston M, Li T, Page MJ, Welch VA (editors). Cochrane Handbook for Systematic Reviews of Interventions version 6.0 (updated July 2019). Cochrane, 2019. Available from www.training.cochrane.org/handbook.

\section{Dreer 2008}

Dreer LE, Elliott TR, Berry J, Fletcher DC, Swanson M, Christopher McNeal J. Cognitive appraisals, distress and disability among persons in low vision rehabilitation. British Journal of Health Psychology 2008;13(Pt 3):449-61.

\section{E 2020a}

E JY, Schrack J, Mihailovic A, West S, Friedman D, Gitlin L, et al. Patterns of daily physical activity across the spectrum of visual field damage in glaucoma. Investigative Ophthalmology and Visual Science 2020;128:1336-1342.

\section{E 2020b}

E JY, Mihailovic A, Kuo PL, West S, Friedman D, Gitlin L, et al. Characterizing the impact of fear of falling on activity and falls in older adults with glaucoma. Journal of the American Geriatrics Society 2020;68:1116-22. [DOI: 10.1111/jgs.16516]

\section{Egger 1997}

Egger M, Davey Smith G, Schneider M, Minder C. Bias in meta-analysis detected by a simple, graphical test. $B M J$ 1997;315(7109):629-634.

\section{Evans 2002}

Evans JR, Fletcher AE, Wormald RP, Ng ES, Stirling S, Smeeth L, et al. Prevalence of visual impairment in people aged 75 years and older in Britain: results from the MRC trial of assessment and management of older people in the community. British Journal of Opthalmology 2002;86(7):795-800.

\section{Gillespie 2012}

Gillespie LD, Robertson MC, Gillespie WJ, Sherrington C, Gates S, Clemson LM, et al. Interventions for preventing falls in older people living in the community. Cochrane Database of Systematic Reviews 2012, Issue 9. Art. No: CD007146. [DOI: 10.1002/14651858.CD007146.pub3]

\section{Glanville 2006}

Glanville JM, Lefebvre C, Miles JN, Camosso-Stefinovic J. How to identify randomized controlled trials in MEDLINE: ten years on. Journal of the Medical Library Association 2006;94(2):130-6.

\section{GRADEpro 2015 [Computer program]}

McMaster University (developed by Evidence Prime) GRADEpro GDT. Hamilton (ON): McMaster University (developed by Evidence Prime), 2015.

\section{Higgins 2019}

Higgins JPT, Savović J, Page MJ, Elbers RG, Sterne JAC. Chapter 8: Assessing risk of bias in a randomized trial. In: Higgins JPT, Thomas J, Chandler J, Cumpston M, Li T, Page MJ, Welch VA (editors). Cochrane Handbook for Systematic Reviews of Interventions version 6.0 (updated July 2019). Cochrane, 2019. Available from www.training.cochrane.org/handbook.

\section{Hoffmann 2014}

Hoffmann TC, Glasziou PP, Boutron I, Milne R, Perera R, Moher D, et al. Better reporting of interventions: template for intervention description and replication (TIDieR) checklist and guide. BMJ 2014;348:g1687.

\section{Holbrook 2011}

Holbrook EA, Stevens SL, Kang M, Morgan DW. Validation of a talking pedometer for adults with visual impairment. Medicine Science Sports and Exercise 2011;43(6):1094-9.

\section{Hopewell 2018}

Hopewell S, Adedire O, Copsey BJ, Boniface GJ, Sherrington C, Clemson L, et al. Multifactorial and multiple component interventions for preventing falls in older people living in the community. Cochrane Database of Systematic Reviews 2018, Issue 7. Art. No: CD012221. [DOI: 10.1002/14651858.CD012221.pub2]

\section{Hutchinson 2018}

Hutchinson M, Wendt N, Smith ST. Trial implementation of a telerehabilitation exercise system in residential aged care. Studies in Health Technology and Informatics 2018;246:62-74. 


\section{Ivers 1998}

Ivers RQ, Cumming RG, Mitchell P, Attebo K. Visual impairment and falls in older adults: The Blue Mountains Eye Study. Journal of the American Geriatrics Society 1998;46(1):58-64.

\section{Kendrick 2014}

Kendrick D, Kumar A, Carpenter H, Zijlstra GAR, Skelton DA, Cook JR, et al. Exercise for reducing fear of falling in older people living in the community. Cochrane Database of Systematic Reviews 2014, Issue 11. Art. No: CD009848. [DOI: 10.1002/14651858.CD009848.pub2]

\section{Klein 2003}

Klein B, Moss S, Klein R, Lee K, Cruickshanks K. Associations of visual function with physical outcomes and limitations 5 years later in an older population: the Beaver Dam Eye Study. Ophthalmology 2003;110(4):644-50.

\section{Lamb 2005}

Lamb SE, Jorstad-Stein EC, Hauer K, Becker C. Development of a common outcome data set for fall injury prevention trials: The Prevention of Falls Network Europe consensus. Journal of the American Geriatrics Society 2005;53(9):1618-22.

\section{Legood 2002}

Legood R, Scuffham P, Cryer C. Are we blind to injuries in the visually impaired? A review of the literature. Injury Prevention 2002;8(2):155-60.

\section{Li 2017}

Li W, Procter-Gray E, Churchill L, Crouter SE, Kane K, Tian J, et al. Gender and age differences in levels, types and locations of physical activity among older adults living in car-dependent neighborhoods. Journal of Frailty and Aging 2017;6(3):129-35.

\section{Markowitz 2006a}

Markowitz SN. Principles of modern low vision rehabilitation. Canadian Journal of Ophthalmology 2006;41(3):289-312.

\section{Markowitz 2006b}

Markowitz M. Occupational therapy interventions in low vision rehabilitation. Canadian Journal of Ophthalmology 2006;41(3):340-7.

\section{McKenzie 2019}

McKenzie JE, Brennan SE, Ryan RE, Thomson HJ, Johnston RV. Chapter 9: Summarizing study characteristics and preparing for synthesis. In: Higgins JPT, Thomas J, Chandler J, Cumpston M, Li T, Page MJ, Welch VA (editors). Cochrane Handbook for Systematic Reviews of Interventions version 6.0 (updated July 2019). Cochrane, 2019. Available from www.training.cochrane.org/handbook.

\section{Mihailovic 2017}

Mihailovic A, Swenor BK, Friedman DS, West SK, Gitlin LN, Ramulu PY. Gait implications of visual field damage from glaucoma. Translational Vision Science and Technology 2017;6(3):23

\section{Murphy 2002}

Murphy SL, Williams CS, Gill TM. Characteristics associated with fear of falling and activity restriction in communityliving older persons. Journal of the American Geriatrics Society 2002;50(3):516-20.

\section{Ong 2018}

Ong SR, Crowston JG, Loprinzi PD, Ramulu PY. Physical activity, visual impairment, and eye disease. Eye 2018;32(8):1296-303.

\section{Pascolini 2012}

Pascolini D, Mariotti SP. Gobal data on visual impairment 2010. British Journal of Ophthalmology 2012;96(5):614-618.

\section{Pelletier 2016}

Pelletier AL, Rojas-Roldan L, Coffin J. Vision loss in older adults. American Family Physician 2016;94(3):219-26.

\section{Preedy 2009}

Preedy VR, Watson RR. Handbook of Disease Burdens and Quality of Life Measures. New York: Springer, 2009.

\section{Ramulu 2009}

Ramulu P. Glaucoma and disability: which tasks are affected, and at what stage of disease? Current Opinion in Ophthalmology 2009;20(2):92-8.

\section{Ramulu 2019}

Ramulu PY, Mihailovic A, West SK, Friedman DS, Gitlin LN. What is a falls risk factor? factors associated with falls per time or per step in individuals with glaucoma. Journal of the American Geriatrics Society 2019;67(1):87-92.

\section{Salive 1994}

Salive ME, Guralnik J, Glynn RJ, Christen W, Wallace RB, Ostfeld AM. Association of visual impairment with mobility and physical function. Journal of the American Geriatrics Society 1994;42(3):287-92.

\section{Schrack 2016}

Schrack JA, Cooper R, Koster A, Shiroma EJ, Murabito JM, Rejeski WJ, et al. Assessing daily physical activity in older adults: unraveling the complexity of monitors, measures, and methods. The Journals of Gerontology. Series A, Biological Sciences and Medical Sciences 2016;71(8):1039-48.

\section{Sherrington 2019}

Sherrington C, Fairhall NJ, Wallbank GK, Tiedemann A, Michaleff ZA, Howard K, et al. Exercise for preventing falls in older people living in the community. Cochrane Database of Systematic Reviews 2019, Issue 1. Art. No: CD012424. [DOI: 10.1002/14651858.CD012424.pub2]

\section{Tinetti 1998}

Tinetti ME, Williams CS. The effect of falls and fall injuries on functioning in community-dwelling older persons. The Journals of Gerontology: Series A, Biological Sciences and Medical Sciences 1998;53(2):M112-9. 


\section{van Nispen 2020}

van Nispen RM, Virgili G, Hoeben M, Langelaan M, Klevering J, Keunen JE, et al. Low vision rehabilitation for better quality of life in visually impaired adults. Cochrane Database of Systematic Reviews 2020, Issue 1. Art. No: CD006543. [DOI: 10.1002/14651858.CD006543.pub2]

\section{Virgili 2010}

Virgili G, Rubin G. Orientation and mobility training for adults with low vision. Cochrane Database of Systematic Reviews 2010, Issue 5. Art. No: CD003925. [DOI: 10.1002/14651858.CD003925.pub3]

\section{Welp 2016}

Welp A, Woodbury PB, McCoy MA, Teutsch SM. Understanding the Epidemiology of Vision Loss and Impairment in the United States. National Academies Press, 2016.

\section{WHO 2001}

World Health Organization. ICIDH-2: International Classification of Functioning, Disability and Health. 1st edition. Geneva: World Health Organization, 2001.

\section{WHO 2007}

World Health Organization. A guide for population-based approaches to increasing levels of physical activity: implementation of the WHO global strategy on diet, physical activity and health. apps.who.int/iris/handle/10665/43612 (accessed 10 June 2020).

\section{WHO 2010}

World Health Organisation. Global recommendations on physical activity for health. www.who.int/dietphysicalactivity/ publications/9789241599979/en/ (accessed 10 June 2020).

\section{WHO 2019}

World Health Organization. ICD-11: International statistical classification of diseases and related health problems: eleventh revision. Geneva: World Health Organization, 2019.

\section{Yardley 2002}

Yardley L, Smith H. A prospective study of the relationship between feared consequences of falling and avoidance of activity in community-living older people. Gerontologist 2002;42(1):17-23.

\section{References to other published versions of this review} Skelton 2011

Skelton DA, Neil F, Ballinger C, Gray L, Palmer S, Howe TE. Environmental and behavioural interventions for reducing physical activity limitation in community dwelling visually impaired older people. Cochrane Database of Systematic Reviews 2011, Issue 7. Art. No: CD009233. [DOI: 10.1002/14651858.CD009233]

\section{Skelton 2013}

Skelton DA, Howe TE, Ballinger C, Neil F, Palmer S, Gray L. Environmental and behavioural interventions for reducing physical activity limitation in community-dwelling visually impaired older people. Cochrane Database of Systematic Reviews 2013, Issue 6. Art. No: CD009233. [DOI: 10.1002/14651858.CD009233.pub2]

\section{CHARACTERISTICS OF STUDIES}

Characteristics of included studies [ordered by study ID]

Adams 2018

\section{Study characteristics}

$\begin{array}{ll}\text { Methods } & \text { Study design: RCT } \\ & \text { Number randomized: } 64 \text { (33 in the intervention group; } 31 \text { in the usual activity group) } \\ & \text { Number analyzed: } 64 \\ & \text { Number of centers: } 2 \\ & \text { Date of first enrollment: January } 2015 \\ & \text { Length of follow-up: } 24 \text { weeks } \\ & \text { Sample size estimation: the authors aimed to recruit a total of } 80 \text { community-living visually impaired } \\ \text { older adults to allow for loss to follow-up. } & \text { Country: UK } \\ \text { Age: mean } 78 \text { years (range } 61 \text { to } 95 \text { years) } \\ \text { Sex: } 39 \% \text { male, } 61 \% \text { female }\end{array}$

Environmental and behavioural interventions for reducing physical activity limitation and preventing falls in older people with visual 26 impairment (Review)

Copyright $\odot 2020$ The Cochrane Collaboration. Published by John Wiley \& Sons, Ltd. 
Adams 2018 (Continued)

Key inclusion criteria: having visual impairment, living in own home, walking independently, physically being able to participant in exercise class

Key exclusion criteria: unable to understand instructions in English, uncontrolled medical diseases, having conditions requiring a specialist exercise program, unable to maintain upright position, no indoor mobility, included in other fall prevention programs

Comparability of baseline characteristics: comparable

Interventions

Behavioral intervention \#1: the exercise program (Falls Management Exercise) consisted of one hour weekly sessions over 12 weeks and was held in community venues with a maximum capacity of ten participants per group. The exercises consisted of balance specific, individually-tailored and targeted training for dynamic balance, strength, endurance, flexibility, gait and functional skills, training to improve 'righting' or 'correcting' skills to avoid a fall and backward-chaining i.e. retraining of the ability to get down to and up from the floor. Functional floor exercises and adapted Tai Chi exercises were also carried out with progressively more challenging content over the 12 weeks. Resistance bands and mats were used. Participants were also advised to exercise at home for up to two hours per week. The exercises were to be performed if possible daily, on the days the participant was not attending the exercise class. All home programs contained 'prompts' that linked exercises to daily tasks e.g. performing heel raises whilst waiting for the kettle to boil, in order to improve adherence. Exercises were provided in a large text booklet, DVD or audio format. Exercises were designed to be completed in 10 to $20 \mathrm{~min}$ blocks, become more challenging and graduate into longer periods.

Control intervention \#2: participants received no intervention and continued with their usual activities. They were offered an equivalent exercise program after the 24 weeks follow-up.

Outcomes Primary outcome: fear of falling scores at 24 weeks measured by The Short Falls Efficacy Scale - International (SFES-I).

Secondary outcomes: (1) physical activity (self-reported physical activity questionnaire [Phone-FITT]), (2) health-related quality of life (European Quality of Life 15), (3) activity avoidance, (4) time Get Up \& Go test, (5) falls risks (Falls Risk Assessment Tool), (6) loneliness (Six-Item Scale for Overall, Emotional, and Social Loneliness), (7) Home Anxiety and Depression Scale (14 items), (8) Work and Social Adjustment Scale.

Notes Funding sources: Public Health Research Programme of the National Institute for Health Research (NIHR), Health Promotion Interventions for People with Impairment Programme, UK

Statistical analyses: appropriate

Subgroup analyses: none reported

Registration: ISRCTN ID: 16949845

\section{Risk of bias}

\begin{tabular}{lll}
\hline Bias & Authors' judgement & Support for judgement \\
\hline $\begin{array}{l}\text { Random sequence genera- } \\
\text { tion (selection bias) }\end{array}$ & Low risk & $\begin{array}{l}\text { Randomization was stratified by center and was administered centrally via } \\
\text { Newcastle Clinical Trials Unit using a secure web based system using a blocked } \\
\text { allocation system to allocate participants to the two groups. }\end{array}$ \\
\hline $\begin{array}{l}\text { Allocation concealment } \\
\text { (selection bias) }\end{array}$ & Low risk & $\begin{array}{l}\text { A blocked allocation (permuted random blocks of variable length) system was } \\
\text { used to allocate participants to the two groups (block size will not be disclosed } \\
\text { to the investigators) in a 1:1 ratio to intervention and control groups. Partic- } \\
\text { ipant screening ID, initials and gender were entered into the web-based sys- } \\
\text { tem, which would return the allocation status. Participants were informed by } \\
\text { telephone, of their allocated treatment group following randomization. }\end{array}$
\end{tabular}


Adams 2018 (Continued)

Blinding of participants High risk Participants were informed of their allocated treatment group following ranand personnel (perfordomization. mance bias)

All outcomes

Blinding of outcome as-
sessment (detection bias) $\quad$ Unclear risk Insufficient information to assess.

All outcomes

Incomplete outcome data Low risk

(attrition bias)

All outcomes
49 out of 64 (92\%) participants completed the 24-week visit (four had withdrawn completely and one was lost to follow up from the study). In two cases, assessments were completed outside the two-week limit, due to other commitments or extenuating circumstances. All participants in the intervention group remaining in the trial at each time point completed each of the questionnaires. There were two occasions when participants in the usual activity group partially completed a questionnaire, and two occasions on which whole questionnaires were not completed.

Selective reporting (re- High risk Not all outcomes reported: i.e., activity avoidance, and loneliness.
porting bias)
porting bias)

\begin{tabular}{ll}
\hline Other bias $\quad$ Low risk $\quad$ No other sources of bias identified. \\
\hline
\end{tabular}

\section{Campbell 2005}

\section{Study characteristics}

Methods

\section{Study design: RCT}

Number randomized: 391 (100 in environmental group, 97 in behavioral group, 98 in the environmental and behavioral group, 96 in the social visit group)

Number analyzed: 391

Number of centers: 2

Date of first enrollment: January 2002

Length of follow-up: 12 months

Sample size estimation: the authors used the rate of falls in those aged $\geq 75,35 \%$ reduction in falls achieved, a power of 0.80 , and a two sided significance of 0.05 . The authors allowed for the Poisson distribution of falls and a $20 \%$ dropout rate.

\section{Participants}

Country: New Zealand

Age: mean 84 years (range 75 to 96 years)

Sex: $32 \%$ male, $68 \%$ female

Key inclusion criteria: having poor vision (visual acuity of $6 / 24$ or worse in the better eye after the best possible correction), and living in the community.

Key exclusion criteria: unable to walk around their own residence, receiving physiotherapy at time of enrollment, could not understand the study requirement

Comparability of baseline characteristics: comparable 
Campbell 2005 (Continued)

Interventions

Environmental intervention \#1: occupational therapists visited the person at home and assessed home safety using a checklist. They identified hazards and initiated a discussion with the participant about any items that could lead to falls. The therapists and participant agreed on which recommendations to implement.The therapists listed these recommendations in a follow-up letter to the participant. They facilitated provision of equipment and payment from usual sources depending on the price and type of the home modification. Referrals were made to the Royal New Zealand Foundation of the Blind. A second home visit was needed to sign off the equipment installed by some providers.

Behavioral intervention \#2:physiotherapists initiated a one-year Otago exercise program (specific muscle strengthening and balance retraining exercises). It was modified for those with severe visual acuity loss, and with vitamin D supplementation. The physiotherapists prescribed the exercises during five home visits at weeks one, two, four, and eight and a booster visit after six months. The degree of difficulty of the exercise and the number of 1,2 , and $3 \mathrm{~kg}$ ankle cuff weights were used for muscle strengthening and increased at each visit as appropriate. Audio tapes of the exercises in four different levels of difficulty were available for those who could not see the exercise instruction sheets. Participants were expected to exercise at least three times a week (about 30 minutes a session) and to walk. The physiotherapists delivered vitamin $D$ tablets if needed. For the months with no scheduled home visit, the physiotherapists telephoned to encourage the person to maintain motivation and discuss any problems.

Environmental and behavioral intervention \#3: received both the environmental and behavioral intervention.

Control intervention \#4: research staff made two home visits lasting an hour each during the first six months of the trial to participants who were not randomized to either environmental or behavioral intervention groups.

Outcomes Primary outcome: number of fallers at 12 months

Secondary outcomes: (1) falls per person year, (2) number of injurious falls, (3) injurious falls per person year, (4) incidence rate ratio for falls, (5) incidence rate ratio for injurious falls.

$\begin{array}{ll}\text { Notes } & \text { Funding sources: Health Research Council of New Zealand } \\ & \text { Statistical analyses: appropriate } \\ & \text { Subgroup analyses: reported } \\ \text { Registration: ISRCTN ID: } 15342873\end{array}$

\section{Risk of bias}

\begin{tabular}{|c|c|c|}
\hline Bias & Authors' judgement & Support for judgement \\
\hline $\begin{array}{l}\text { Random sequence genera- } \\
\text { tion (selection bias) }\end{array}$ & Low risk & $\begin{array}{l}\text { A statistician developed the group allocation schedule using computer gener- } \\
\text { ated random numbers }\end{array}$ \\
\hline $\begin{array}{l}\text { Allocation concealment } \\
\text { (selection bias) }\end{array}$ & Low risk & $\begin{array}{l}\text { The schedule was held by an independent person at a separate site and was } \\
\text { accessed by a research administrator for the study, who telephoned after each } \\
\text { baseline assessment was completed }\end{array}$ \\
\hline $\begin{array}{l}\text { Blinding of participants } \\
\text { and personnel (perfor- } \\
\text { mance bias) } \\
\text { All outcomes }\end{array}$ & High risk & $\begin{array}{l}\text { Participants were informed of their allocated treatment group following ran- } \\
\text { domization. }\end{array}$ \\
\hline $\begin{array}{l}\text { Blinding of outcome as- } \\
\text { sessment (detection bias) } \\
\text { All outcomes }\end{array}$ & Low risk & $\begin{array}{l}\text { Falls were monitored for one year for each person using prepaid, addressed, } \\
\text { tear-off monthly postcard calendars. The independent assessors in each cen- } \\
\text { ter telephoned participants to record the circumstances of the falls and any re- } \\
\text { sulting injuries or use of resources. They remained blind to group allocation. }\end{array}$ \\
\hline
\end{tabular}

Environmental and behavioural interventions for reducing physical activity limitation and preventing falls in older people with visual 


\section{Campbell 2005 (Continued)}

Incomplete outcome data Low risk (attrition bias)

All outcomes
361 out of 391 (92\%) participants completed one year of follow-up. The mean and total follow-up time were equal by four arms. Reasons for not being follow-up were reported and similar across four arms.

Selective reporting (re- Low risk Outcomes specified in the study protocol were reported. porting bias)

Other bias Low risk No other sources of bias identified.

\section{Study characteristics}

\section{Methods}

Study design: RCT

Number randomized: 120 (60 in the intervention group; 60 in the usual activity group)

Number analyzed: 120

Number of centers: 1

Date of first enrollment: August 2010

Length of follow-up: 3 months

Sample size estimation: the study was powered to measure the impact of the Alexander technique on physical function such that 60 individuals in each of the two groups $(\mathrm{N}=120)$ would give the study $80 \%$ power to detect a $15 \%$ between-group difference at a $5 \%$ level of significance allowing for $15 \%$ dropouts during the 12 months for the primary outcome.

Participants

Country: Australia

Age: mean 75 years $(S D=10)$

Sex: $29 \%$ male, $71 \%$ female

Key inclusion criteria: having a vision impairment, having had an orientation and mobility program from Guide Dogs within last five years, living within Syndey metropolitan area, not need an interpreter

Key exclusion criteria: not reported

Comparability of baseline characteristics: comparable

Interventions

Behavioral intervention \#1: participants received 12 lessons (30 minutes long) for Alexander technique in individual one-on-one sessions in the participant's own home. The Alexander technique was a physical conditioning program designed to alter and improve the way individuals move and react to physical stimuli. A typical session included completing activities of daily living, while being assisted by a trained Alexander technique practitioner. The completion of these activities was accompanied by psychological techniques including mindfulness, co-ordination and body-mapping. The exercises were designed to improve postural stability, co-ordination and confidence during movement. A lesson protocol was developed using everyday activities such as movements between sitting and standing, getting to and from the floor, and walking, climbing stairs and conducting everyday activities. Subsequent lessons were based on prior progress, and the lesson plan was modified as necessary. The Alexander Technique lessons were delivered by one person who was an accredited teacher of the Alexander Technique.

Control intervention \#2: participants received usual care (able to access orientation and mobility programs) from Guide Dogs. 
Gleeson 2017 (Continued)

Outcomes

Primary outcome: physical performance from the Short Physical Performace Battery at 12 months

Secondary outcomes: (1) number of falls, (2) falls rate per person, (3) fear of falling scores by the Short Falls Efficacy Scale - International (SFES-I), (4) mood with the Geriatric Depression Scale-5 (GDS-5) and the Positive and Negative Affect Scale (PANAS), (5) the Perceived Visual Ability Scale, (6) the Keele Assessment of Participation

Notes

Funding sources: Guide Dogs NSW/ACT, Sydney, Australia; The Australian Society of Teachers of the Alexander Technique, Beechworth, Victoria; and the FM Alexander Trust, London, UK

Statistical analyses: appropriate

Subgroup analyses: reported

Registration: ACTRN12610000634077

\title{
Risk of bias
}

\begin{tabular}{|c|c|c|}
\hline Bias & Authors' judgement & Support for judgement \\
\hline $\begin{array}{l}\text { Random sequence genera- } \\
\text { tion (selection bias) }\end{array}$ & Low risk & $\begin{array}{l}\text { A block randomized (block permutation size } 1,2 \text { and } 4 \text { ) sequence using a com- } \\
\text { puter generated list. }\end{array}$ \\
\hline $\begin{array}{l}\text { Allocation concealment } \\
\text { (selection bias) }\end{array}$ & Low risk & $\begin{array}{l}\text { The computer generated list was kept by a separate center-based investigator } \\
\text { who had no contact with the participants. }\end{array}$ \\
\hline $\begin{array}{l}\text { Blinding of participants } \\
\text { and personnel (perfor- } \\
\text { mance bias) } \\
\text { All outcomes }\end{array}$ & High risk & $\begin{array}{l}\text { The participants and the intervention providers could not be masked to group } \\
\text { allocation. }\end{array}$ \\
\hline $\begin{array}{l}\text { Blinding of outcome as- } \\
\text { sessment (detection bias) } \\
\text { All outcomes }\end{array}$ & Low risk & $\begin{array}{l}\text { All outcome assessors remained masked to group allocation for all assess- } \\
\text { ments, and participants were asked not to reveal their allocation status. }\end{array}$ \\
\hline $\begin{array}{l}\text { Incomplete outcome data } \\
\text { (attrition bias) } \\
\text { All outcomes }\end{array}$ & Unclear risk & $\begin{array}{l}\text { Of the } 120 \text { participants who entered the study, } 10 \text { did not complete all assess- } \\
\text { ments. Data not reported for these people. Since } 92 \% \text { participants were fol- } \\
\text { lowed up, we judged it as unclear risk of bias. }\end{array}$ \\
\hline $\begin{array}{l}\text { Selective reporting (re- } \\
\text { porting bias) }\end{array}$ & Low risk & Outcomes specified in the study protocol were reported. \\
\hline Other bias & Low risk & No other sources of bias identified. \\
\hline
\end{tabular}

Jeter 2015

\section{Study characteristics}

Methods

\author{
Study design: RCT \\ Number randomized: 21 (11 in the intervention group; 10 in the usual activity group) \\ Number analyzed: 17 \\ Number of centers: 1 \\ Date of first enrollment: October 2012
}


Jeter 2015 (Continued)

Length of follow-up: 8 weeks

Sample size estimation: the author reported a sample size calculation was not applicable to this type of exploratory study; however, a minimum of 10 subjects per group was feasible to provide an indication of the acceptability.

\begin{tabular}{|c|c|}
\hline \multirow[t]{6}{*}{ Participants } & Country: US \\
\hline & Age: median 59 years (range: 27 to 85 years) \\
\hline & Sex: $35 \%$ male, $65 \%$ female \\
\hline & $\begin{array}{l}\text { Key inclusion criteria: blindness (best-corrected visual acuity worse than } 20 / 200 \text { and/or visual field less } \\
\text { than } 20^{\circ} \text { in diameter in the better eye), ocular diseases that were stable throughout 3-6 months, able to } \\
\text { participate in yoga program, English-speaking }\end{array}$ \\
\hline & $\begin{array}{l}\text { Key exclusion criteria: individuals with vestibular disorders, acute orthopaedic problems that affect } \\
\text { ambulation, history of neurologic disease (e.g. peripheral neuropathy), or who were pregnant or taking } \\
\text { medication that could affect balance (e.g. sleeping pills) }\end{array}$ \\
\hline & Comparability of baseline characteristics: comparable \\
\hline \multirow[t]{2}{*}{ Interventions } & $\begin{array}{l}\text { Behavioral intervention \#1: Ashtanga-based yoga therapy (AYT) for } 8 \text { weeks. One group session per } \\
\text { week with the instructor and an experienced yoga assistant. Participants were provided with a free yo- } \\
\text { ga mat and an audio CD developed by the author to practice at home and were asked to practice at } \\
\text { least twice a week (i.e. equivalent to approximately } 16 \text { home practice sessions during the intervention } \\
\text { period). The AYT is amenable to study because it is composed of a standardized sequence of postures } \\
\text { held for a fixed duration. Each pose was held for five breaths or for as long as the subject was able. }\end{array}$ \\
\hline & Control intervention \#2: waitlist group with no active intervention. \\
\hline \multirow[t]{2}{*}{ Outcomes } & Primary outcome: center of pressure at 8 weeks, stability index at 8 weeks \\
\hline & $\begin{array}{l}\text { Secondary outcomes: (1) timed one-leg stance, (2) physical function, (3) Illinois fear of falling scores } \\
\text { (only P-value reported). }\end{array}$ \\
\hline \multirow[t]{4}{*}{ Notes } & $\begin{array}{l}\text { Funding sources: Louise L. Sloan Research Grant Award, Lions Vision Research Foundation, Wilmer Eye } \\
\text { Institute, Johns Hopkins University }(\$ 2,000) \text { and National Eye Institute, Diversity Supplement. }\end{array}$ \\
\hline & Statistical analyses: appropriate \\
\hline & Subgroup analyses: not reported \\
\hline & Registration: NCT01366677 \\
\hline
\end{tabular}

\section{Risk of bias}

\section{Bias \\ Authors' judgement Support for judgement}

Random sequence genera- Low risk tion (selection bias)
Randomization to group assignment was conducted by the study PI using the random number generator in MATLAB (Mathworks, Inc.).

\begin{tabular}{lll}
\hline $\begin{array}{l}\text { Allocation concealment } \\
\text { (selection bias) }\end{array}$ & Low risk & $\begin{array}{l}\text { A research assistant assigned unidentifiable subject IDs (i.e. \#1-21) to subjects } \\
\text { after enrollment. }\end{array}$ \\
\hline $\begin{array}{l}\text { Blinding of participants } \\
\begin{array}{l}\text { and personnel (perfor- } \\
\text { mance bias) }\end{array}\end{array}$ & High risk & Masking participants to the yoga intervention was not possible. \\
$\begin{array}{l}\text { All outcomes } \\
\begin{array}{l}\text { Blinding of outcome as- } \\
\text { sessment (detection bias) }\end{array}\end{array}$ & Low risk & $\begin{array}{l}\text { Trained research assistants were masked to the group assignment during data } \\
\text { collection. }\end{array}$ \\
\hline
\end{tabular}

Environmental and behavioural interventions for reducing physical activity limitation and preventing falls in older people with visual 
Jeter 2015 (Continued)

All outcomes

Incomplete outcome data Low risk (attrition bias)

All outcomes
17 of 21 (81\%) participants completed 8-week of follow-up. Two participants in each arm were not followed up, and reasons for not being followed up were reported and similar.

\section{Selective reporting (re- High risk} porting bias)

The data represented the quantitative results that are a subset of a larger battery of assessments that included psychological questionnaires and other qualitative information

Other bias Low risk No other sources of bias identified.

Kovacs 2012

\section{Study characteristics}

Study design: RCT
Number randomized: 41 (21 in the intervention group; 20 in the usual activity group)
Number analyzed: 41
Number of centers: 1
Date of first enrollment: February 2010
Length of follow-up: 8 weeks
Sample size estimation: the author reported this study had small sample size which reduced the statis-
tical power of their analyses. Based on their results, a post hoc power analysis indicated that at least
171 participants in each group would require to achieve a statistical power of $80 \%$.

Participants Country: Hungary

Age: mean 70 years (SD: 7 years)

Sex: $100 \%$ female

Key inclusion criteria: living in nursing homes with age-related visual impairment, being female.

Key exclusion criteria: being totally blind, had lived in the nursing home for less than 2 months, being unable to walk around their own residence, progressively being unable to walk around their own residence, progressively increasing severity of neurological, and unstable cardiovascular diseases that would limit participation in exercise program, planned moving away from the nursing home during the study period and participated in an exercise program including balance exercise within 6 months

Comparability of baseline characteristics: comparable

Interventions

Behavioral intervention \#1: participants joined twice a week in a multimodal exercise program for 30 min and twice a week in the standard osteoporosis exercise program. The multimodal exercise program included balance and strength exercises based on Otago Exercise Program.

Control intervention \#2: participants joined in the standard osteoporosis exercise program alone for four times a week in $30 \mathrm{~min}$.

Outcomes

Primary outcome: Berg Balance Scale at 6 months,

Secondary outcomes: (1) Timed Up and Go, (2) Barthel activity of daily living, (3) number of fallers, (4) mean length of time to first fall in weeks. 
Kovacs 2012 (Continued)

Notes
Funding sources: the study was not sponsored

Statistical analyses: appropriate

Subgroup analyses: not reported

Registration: not available

\section{Risk of bias}

\begin{tabular}{|c|c|c|}
\hline Bias & Authors' judgement & Support for judgement \\
\hline $\begin{array}{l}\text { Random sequence genera- } \\
\text { tion (selection bias) }\end{array}$ & Low risk & Blocked randomization was performed (with a block size of 4). \\
\hline $\begin{array}{l}\text { Allocation concealment } \\
\text { (selection bias) }\end{array}$ & Low risk & $\begin{array}{l}\text { Assignment was numbered opaque identical sealed envelopes. The envelopes } \\
\text { were prepared by an independent physiotherapist. }\end{array}$ \\
\hline $\begin{array}{l}\text { Blinding of participants } \\
\text { and personnel (perfor- } \\
\text { mance bias) } \\
\text { All outcomes }\end{array}$ & High risk & Therapist and participants were not blinded to group allocation. \\
\hline $\begin{array}{l}\text { Blinding of outcome as- } \\
\text { sessment (detection bias) } \\
\text { All outcomes }\end{array}$ & Low risk & $\begin{array}{l}\text { The outcome assessor was uninformed about group allocation and was not in- } \\
\text { volved in proceedings of the exercise programs. Participants were asked not to } \\
\text { reveal details of their treatment to the outcome assessor. }\end{array}$ \\
\hline $\begin{array}{l}\text { Incomplete outcome data } \\
\text { (attrition bias) } \\
\text { All outcomes }\end{array}$ & Low risk & $\begin{array}{l}\text { All participants completed 8-week of follow-up. No participants were excluded } \\
\text { from the study. }\end{array}$ \\
\hline $\begin{array}{l}\text { Selective reporting (re- } \\
\text { porting bias) }\end{array}$ & Low risk & Outcomes specified in the study protocol were reported \\
\hline Other bias & Low risk & No other sources of bias identified \\
\hline
\end{tabular}

Waterman 2016

\section{Study characteristics}

Study design: RCT
Number randomized: 49 (16 in environmental group; 17 in environmental and behavior group, 16 in the
control group)
Number analyzed: 43
Number of centers: 1
Date of first enrollment: July 2011
Length of follow-up: 6 months
Sample size estimation: the author reported the intended sample size was 30 participants in each
group (a simple randomization 1:1:1 ratio) allowing for $10 \%$ attrition rate.

Participants Country: UK

Environmental and behavioural interventions for reducing physical activity limitation and preventing falls in older people with visual 
Waterman 2016 (Continued)

Age: mean 81 years (SD: 8 years)

Sex: $35 \%$ male, $65 \%$ female

Key inclusion criteria: having a vision impairment (Binocular visual acuity $>0.6$ LogMAR, and/or moderate visual field loss defined as affecting more than $20 \%$ of the test locations used in a binocular Esterman test), living independently in community, being able to walk around own residence, cognitively able to participate and understand study requirements.

Key exclusion criteria: receiving an OT or physiotherapist intervention or home safety assessment and modification or exercise intervention, including attendance at a Falls Clinic, not achieving between 7 and 10 on the Abbreviated Mental Test.

Comparability of baseline characteristics: comparable

Interventions

Environmental intervention \#1: participants received the Home Safety (HS) program implemented by an Occupational Therapist (OT). An experienced trained OT used an amended version of the Westmead Home Safety Assessment to discuss environmental hazards present in their homes with participants. This resulted in a jointly agreed action plan incorporating participant needs and views. The action plan focused on how the participant could alter their environment to reduce the likelihood of falls.

Environmental and behavioral intervention \#2: participants received the HS plus the home exercise programs both implemented by the OT and supported by a volunteer peer mentor. The HS was described above, the home exercise included the Otago Exercise Programme (OEP) for 6 months. The exercises, stressing both strength and balance, were individually prescribed, progress in difficulty, and were undertaken for $30 \mathrm{~min}$ at least three times per week. A walking plan was also agreed with all participants to be undertaken at least twice per week.

Control intervention \#3: usual care from the NHS, but in addition received three social visits and two telephone calls by lay visitors (volunteer student nurses, alumni and members of staff from the university)

Outcomes

Primary outcome: number of falls at 6 months, number of injurious falls at 6 months.

Secondary outcomes: (1) adherence rate, (2) self-reported physical activity questionnaire (Phone-FITT), (3) objective measures of physical activity (activPALTM activity monitor): daily step counts and walking time (minutes) (4) quality of life (12-Item Short Form Health Survey), (5) visual disability (Vision-Related Quality of Life), (6) attitudes to Falls-Related Interventions Scale, (7) fear of falling (SFES-I).

Notes

Funding sources: National Institute for Health Research (NIHR) under the Research for Patient Benefit Programme (RfPB), reference number: PB-PG-0909-20090.

Statistical analyses: appropriate

Subgroup analyses: not reported

Registration: ISRCTN53433311

\section{Risk of bias}

\begin{tabular}{lll}
\hline Bias & Authors' judgement & Support for judgement \\
\hline $\begin{array}{l}\text { Random sequence genera- } \\
\text { tion (selection bias) }\end{array}$ & Low risk & $\begin{array}{l}\text { Participants were then independently randomized by the Clinical Trials Unit } \\
\text { via a web-based secure randomization service, which informed the Occupa- } \\
\text { tional Therapist or lay visitor coordinator of the group assignment }\end{array}$ \\
\hline $\begin{array}{l}\text { Allocation concealment } \\
\text { (selection bias) }\end{array}$ & Low risk & $\begin{array}{l}\text { Occupational Therapist and coordinator did not know the next assignment } \\
\text { when implementing the randomization }\end{array}$ \\
\hline $\begin{array}{l}\text { Blinding of participants } \\
\text { and personnel (perfor- } \\
\text { mance bias) }\end{array}$ & High risk & $\begin{array}{l}\text { Participants and the occupational therapist delivering the intervention, social } \\
\text { visitors and peer mentors could not be blinded to group allocation. }\end{array}$ \\
\hline
\end{tabular}

Environmental and behavioural interventions for reducing physical activity limitation and preventing falls in older people with visual 
Waterman 2016 (Continued)

All outcomes

\begin{tabular}{lll}
$\begin{array}{l}\text { Blinding of outcome as- } \\
\text { sessment (detection bias) } \\
\text { All outcomes }\end{array}$ & Low risk & The research assistant and statistician were blinded to group allocation \\
\hline $\begin{array}{l}\text { Incomplete outcome data } \\
\text { (attrition bias) }\end{array}$ & Low risk & $\begin{array}{l}43 \text { of } 49(88 \%) \text { participants completed } 6 \text {-month of follow-up. Number of partic- } \\
\text { ipants with follow-up were equal between groups. No missing outcome data. } \\
\text { Participants were phoned to ensure complete data set. }\end{array}$
\end{tabular}

\begin{tabular}{lll}
\hline $\begin{array}{l}\text { Selective reporting (re- } \\
\text { porting bias) }\end{array}$ & Low risk & Outcomes specified in the study protocol were reported \\
\hline Other bias & Low risk & No other sources of bias identified \\
\hline
\end{tabular}

FaME: Falls Management Exercise

Phone-FITT: Telephone questionnaire for self-report of physical activity

SFES-I: Short Falls Efficacy Scale-International

EuroQoL: European Quality of Life

PANAS: Positive and Negative Affect Scale

GDS-5: Geriatric Depression Scale-5

Ashtanga-based yoga therapy (AYT)

OT: Occupational Therapist

HS: Home Safety

OEP: Otago Exercise Programme

SF-12: 12-Item Short Form Health Survey

Characteristics of excluded studies [ordered by study ID]

\begin{tabular}{ll}
\hline Study & Reason for exclusion \\
\hline Balliet 1985 & Not the comparison of interest: visual field training \\
\hline Cumming 2007 & $\begin{array}{l}\text { Not the comparison of interest: Aim of intervention was not to decrease physical activity limitation } \\
\text { and no physical activity outcome measures. Most had change in glasses, with only a few having an } \\
\text { occupational therapy Intervention }\end{array}$ \\
\hline Dahlin-Ivanoff 2002 & $\begin{array}{l}\text { Outcomes of interest not measured. Outcome measure was perceived security in the performance } \\
\text { of daily occupations rather than a subjective or objective measure of physical activity or change in } \\
\text { physical activity }\end{array}$ \\
\hline
\end{tabular}

\begin{tabular}{ll}
\hline Day 2002 & Not the comparison of interest \\
\hline de Hann 2016 & Not the comparison of interest \\
\hline Deemer 2017 & Outcomes of interest not measured \\
\hline Deremeik 2007 & Outcomes of interest not measured:outcomes were rehabilitation goals and visual correction. \\
\hline Dillon 2018 & Not a randomized or quasi-randomized controlled trial: protocol development \\
\hline Elliott 2016 & Outcomes of interest not measured \\
\hline Girdler 2010 & Not the comparison of interest: aimed to increase participation in life situations \\
\hline Gleeson 2014 & Not a randomized or quasi-randomized controlled trial \\
\hline
\end{tabular}

Environmental and behavioural interventions for reducing physical activity limitation and preventing falls in older people with visual 


\begin{tabular}{|c|c|}
\hline Study & Reason for exclusion \\
\hline Gutman 2002 & Not a randomized or quasi-randomized controlled trial \\
\hline Hollands 2013 & Not the population of interest \\
\hline Hollands 2015 & Not the population of interest \\
\hline Holloway 2018 & Not the comparison of interest \\
\hline Keay 2018 & Not a randomized or quasi-randomized controlled trial \\
\hline Kuyk 2008 & Not a randomized or quasi-randomized controlled trial \\
\hline Kuyk 2010 & Not the comparison of interest \\
\hline La Grow 2004 & Not a randomized or quasi-randomized controlled trial \\
\hline Machado 2014 & Not a randomized or quasi-randomized controlled trial: \\
\hline McCabe 2000 & Not a randomized or quasi-randomized controlled trial \\
\hline NCT00545220 & Outcomes of interest not measured \\
\hline NCT00769015 & $\begin{array}{l}\text { Outcomes of interest not measured: primary outcome was depressive disorder based on the Pa- } \\
\text { tient Health Questionnaire-9 }\end{array}$ \\
\hline NCT04066075 & Outcomes of interest not measured: primary outcome was physical function \\
\hline NCT04084587 & Not a randomized or quasi-randomized controlled trial \\
\hline Ni 2012 & Not a randomized or quasi-randomized controlled trial \\
\hline Overbury 1996 & Not comparison of interest: perceptual learning training - paper-based \\
\hline Pankow 2004 & Outcomes of interest not measured \\
\hline Rees 2015 & Outcomes of interest not measured \\
\hline Reeves 2004 & Outcomes of interest not measured \\
\hline Renaud 2013 & Not a randomized or quasi-randomized controlled trial \\
\hline Renieri 2013 & Outcomes of interest not measured \\
\hline Riazi 2014 & Not comparison of interest: structured psychological improvement \\
\hline Ribeiro 2015 & Not a randomized or quasi-randomized controlled trial \\
\hline Roets Merken 2015 & Not a randomized or quasi-randomized controlled trial \\
\hline Roqueta 2016 & Not a randomized or quasi-randomized controlled trial \\
\hline Rottman Sagebiel 2017 & Not the population of interest \\
\hline Rubin 2017 & Outcomes of interest not measured \\
\hline
\end{tabular}




\begin{tabular}{|c|c|}
\hline Study & Reason for exclusion \\
\hline Rumrill 1999 & Not the comparison of interest \\
\hline Sand 2016 & Not the population of interest \\
\hline Scanlan 2004 & Not the comparison of interest: extend reading time \\
\hline Sekeryapan 2013 & Not a randomized or quasi-randomized controlled trial \\
\hline Shi 2013 & Not a randomized or quasi-randomized controlled trial \\
\hline Shi 2016 & Not the population of interest \\
\hline Siantar 2015 & Not a randomized or quasi-randomized controlled trial \\
\hline Skalska 2013 & Not a randomized or quasi-randomized controlled trial \\
\hline Skelton 2016 & Outcomes of interest not measured: outcomes were fear of falling and quality of life \\
\hline Smith 2009 & Outcomes of interest not measured \\
\hline Smith 2017 & Not a randomized or quasi-randomized controlled trial \\
\hline Stelmack 2005 & Outcomes of interest not measured: primary outcome was visual function \\
\hline Sturrock 2015 & Not a randomized or quasi-randomized controlled trial \\
\hline Vukicevic 2009 & Not the comparison of interest: eccentric viewing computerized training \\
\hline Wang 2012 & Not a randomized or quasi-randomized controlled trial \\
\hline West 2004 & Outcomes of interest not measured \\
\hline Zijlstra 2009 & Outcomes of interest not measured \\
\hline
\end{tabular}

$\mathrm{RCT}$ : randomized controlled trial

Characteristics of ongoing studies [ordered by study ID]

\section{ACTRN12607000399493}

\begin{tabular}{ll}
\hline Study name & $\begin{array}{l}\text { A randomized controlled trial of a low vision self management program on quality of life in people } \\
\text { with low vision. }\end{array}$ \\
\hline Methods & Study design: RCT \\
& Number randomized: 240 (target) \\
& Number analyzed: not reported \\
& Number of centers: not reported \\
& Date of first enrollment: January 2007 \\
& Length of follow-up: 16 months \\
& Sample size estimation: not reported
\end{tabular}


ACTRN12607000399493 (Continued)
Participants
Country: Australia
Age: not reported
Sex: not reported
Key inclusion criteria: aged 55 or older, visual impairment (visual acuity of $<6 / 12$ and $>6 / 480$ in the better eye with habitual correction), English-speaking, no cognitive impairment, adequate hearing.
Key exclusion criteria: not reported
Comparability of baseline characteristics: not applicable

Interventions

Behavioral intervention \#1: participants receive new low vision self-management program "Living with Low Vision". It consist of eight 3-hour weekly facilitated group sessions. The program is structured and a facilitator manual clearly outline the content and delivery. As well as providing information, the topics in the program are covered by exploring participants' experiences, difficulties and solutions. Participants are encouraged to draw on their extensive life experience and coping mechanisms and to develop new skills and strategies and apply these new techniques in their daily life. Participants are given the option to bring a relative, friend or acre to the program with them.

Control intervention \#2: participants continue to access usual low vision rehabilitation services.

Outcomes

Primary outcome: Vision Impairment Questionnaire (IVI) to assess the restriction of participation in daily activities in people with low vision, the Depression Anxiety Stress Scale (DASS) to assess psychological well-being.

Secondary outcomes: (1) Adaptation to Age-related Vision Loss Scale (AVL-12), (2) The General SelfEfficacy Scale (GSES), (3) The Health Education Impact Questionnaire (HEIQ) scale, (4) The Positive and Negative Affect Schedule (PANAS).

\begin{tabular}{ll}
\hline Starting date & $1 / 03 / 2007$ \\
\hline Contact information & Professor Jill Keeffe \\
\hline Notes & The trial was recruiting participants, accessed on 01/31/2020, through: \\
& http://apps.who.int/trialsearch/Trial3.aspx?trialid=ACTRN12607000399493. \\
\hline
\end{tabular}

IVI: Vision Impairment Questionnaire DASS: Depression Anxiety Stress Scale AVL-12: Adaptation to Age-related Vision Loss Scale GSES: General Self-Efficacy Scale HEIQ: Health Education Impact Questionnaire PANAS: Positive and Negative Affect Scale

\section{A P P E N DICES}

\section{Appendix 1. CENTRAL search strategy}

\#1 MeSH descriptor Vision Disorders \#2 MeSH descriptor Visually Impaired Persons \#3 (low* or handicap ${ }^{\star}$ or subnormal* or impair $^{\star}$ or partial* or disab*) near/3 (vision or visual* or sight*) \#4 (\#1 OR \#2 OR \#3) \#5 MeSH descriptor Rehabilitation \#6 (rehabilitat* or assess ${ }^{\star}$ ) near/4 (low vision) \#7 MeSH descriptor Activities of Daily Living \#8 MeSH descriptor Risk Assessment \#9 MeSH descriptor Risk Factors 
\#10 MeSH descriptor Risk Management

\#11 MeSH descriptor Safety Management

\#12 (home near/3 safet ${ }^{\star}$ )

$\# 13$ (hazard*) near/3 (home or environment ${ }^{\star}$ )

\#14 MeSH descriptor Home Care Services

\#15 MeSH descriptor Occupational Therapy

\#16 MeSH descriptor Exercise Therapy

\#17 MeSH descriptor Physical Therapy Modalities

\#18 behavio* near/3 modif*

\#19 (program*) near/3 (home or exercise* or modif*)

\#20 MeSH descriptor Cognitive Therapy

\#21 MeSH descriptor Behavior Therapy

\#22 (\#5 OR \#6 OR \#7 OR \#8 OR \#9 OR \#10 OR \#11 OR \#12 OR \#13 OR \#14 OR \#15 OR \#16 OR \#17 OR \#18 OR \#19 OR \#20 OR \#21)

\#23 (\#4 AND \#22)

\section{Appendix 2. MEDLINE Ovid search strategy}

1. randomized controlled trial.pt.

2. (randomized or randomised).ab,ti.

3. placebo.ab,ti.

4. dt.fs.

5. randomly.ab,ti.

6. trial.ab,ti.

7. groups.ab,ti.

8. or/1-7

9. exp animals/

10. exp humans/

11. 9 not (9 and 10)

12. 8 not 11

13. exp vision disorders/

14. exp visually impaired persons/

15. ((low\$ or handicap\$ or subnormal\$ or impair\$ or partial\$ or disab\$) adj3 (vision or visual\$ or sight\$)).tw.

16. or/13-15

17. exp rehabilitation/

18. ((rehabilitat\$ or assess\$) adj4 low vision).tw.

19. exp activities of daily living/

20. risk assessment/

21. risk factors/

22. risk management/

23. safety management/

24. (home adj3 safety\$).tw.

25. (hazard\$ adj3 (home or environment\$)).tw.

26. home care services/

27. occupational therapy/

28. exercise therapy/

29. physical therapy modalities/

30. (behavio $\$$ adj3 modif\$).tw.

31. (program $\$$ adj3 (home or exercise $\$$ or modif\$)).tw.

32. Cognitive Therapy/

33. Behavior Therapy/

34. or/17-33

35. 16 and 34

36. 12 and 35

The search filter for trials at the beginning of the MEDLINE strategy is from the published paper by Glanville 2006.

\section{Appendix 3. EMBASE Ovid search strategy}

1. exp randomized controlled trial/

2. exp randomization/

3. exp double blind procedure/

4. exp single blind procedure/

5. random\$.tw. 
6. or/1-5

7. (animal or animal experiment).sh.

8. human.sh.

9. 7 and 8

10.7 not 9

11.6 not 10

12. exp clinical trial/

13. (clin\$ adj3 trial\$).tw.

14. ((singl\$ or doubl\$ or trebl\$ or tripl\$) adj3 (blind\$ or mask\$)).tw.

15. exp placebo/

16. placebo\$.tw.

17. random $\$$.tw.

18. exp experimental design/

19. exp crossover procedure/

20. exp control group/

21. exp latin square design/

22. or/12-21

23. 22 not 10

24. 23 not 11

25. exp comparative study/

26. exp evaluation/

27. exp prospective study/

28. (control\$ or prospectiv\$ or volunteer\$).tw.

29. or $/ 25-28$

30.29 not 10

31.30 not ( 11 or 23 )

32. 11 or 24 or 31

33. exp vision disorder/

34. exp visual impairment/

35. ((low\$ or handicap\$ or subnormal\$ or impair\$ or partial\$ or disab\$) adj3 (vision or visual\$ or sight\$)).tw.

36. or/33-35

37. exp rehabilitation/

38. ((rehabilitat\$ or assess\$) adj4 low vision).tw.

39. exp daily life activities/

40. risk assessment/

41. risk factor/

42. exp home safety/

43. (home adj3 safety\$).tw.

44. exp falling/

45. (hazard\$ adj3 (home or environment\$)).tw.

46. home care/

47. occupational therapy/

48. kinesiotherapy/

49. exp physiotherapy/

50. (behavio $\$$ adj3 modif\$).tw.

51. (program\$ adj3 (home or exercise\$ or modif\$)).tw.

52. Cognitive Therapy/

53. Behavior Therapy/

54. or/37-53

55.36 and 54

56.32 and 55

\section{Appendix 4. CINAHL EBSCO search strategy}

S42 S40 and S41

S41 (MH "Aged+") OR (MH "Aged, 80 and Over") OR (MH "Frail Elderly")

S40 S12 and S39

S39 S20 and S38

$\mathrm{S} 38 \mathrm{~S} 21$ or S22 or S23 or S24 or S25 or S26 or S27 or S28 or S29 or S30 or S31 or S32 or S33 or S34 or S35 or S36 or S37

S37 (MH "Behavior Therapy+") OR (MH "Behavior Therapy (Iowa NIC) (Non-Cinahl)")

S36 (MH "Cognitive Therapy") OR (MH "Cognitive Therapy (lowa NIC) (Non-Cinahl)")

S35 TX ((program* n3 home) or (program* n3 exercise) or (program* n3 modif*))

Environmental and behavioural interventions for reducing physical activity limitation and preventing falls in older people with visual 
S34 TX behavio* n3 modif*

S33 (MH "Physical Therapy")

S32 (MH "Exercise Therapy: Ambulation (lowa NIC)") OR (MH "Exercise Therapy: Balance (Iowa NIC)")

S31 (MH "Occupational Therapy+")

S30 (MH "Home Health Care")

S29 TX ((hazard ${ }^{\star}$ n3 home) or (hazard* n3 environment $\left.\left.{ }^{\star}\right)\right)$

S28 TX home $n 3$ safety*

S27 (MH "Risk Management") OR (MH "Risk Management (lowa NIC) (Non-Cinahl)")

S26 (MH "Risk Factors")

S25 (MH "Risk Assessment") OR (MH "Fall Risk Assessment Tool")

S24 (MH "Activities of Daily Living+") OR (MH "Activities of Daily Living (Saba CCC)") OR (MH "Activities of Daily Living Alteration (Saba CCC)") OR (MH "Instrumental Activities of Daily Living (Saba CCC)") OR (MH "Instrumental Activities of Daily Living Alteration (Saba CCC)") OR (MH "Altered Activities of Daily Living (NANDA) (Non-Cinahl)") OR (MH "Self Care: Activities of Daily Living (lowa NOC)") OR (MH "Self-Care: Instrumental Activities of Daily Living (lowa NOC)")

S23 TX assess* ${ }^{\star} 4$ vision*

S22 TX rehabilitat* $n 4$ vision*

S21 (MH "Rehabilitation of Vision Impaired+")

$\mathrm{S} 20 \mathrm{~S} 13$ or S14 or S15 or S16 or S17 or S18 or S19

S19 TX ((disab* n3 vision) or (disab* n3 visual*) or (disab* $n 3$ sight))

S18 TX ((partial ${ }^{\star} n 3$ vision) or ( partial $^{\star} n 3$ visual $\left.^{\star}\right)$ or (partial ${ }^{\star} n 3$ sight $\left.)\right)$

S17 TX ((impair* n3 vision) or (impair* n3 visual $\left.{ }^{*}\right)$ or (impair ${ }^{\star} n 3$ sight))

S16 TX ((subnormal ${ }^{\star}$ n3 vision) or (subnormal ${ }^{\star}$ n3 visual $\left.{ }^{\star}\right)$ or (subnormal ${ }^{\star}$ n3 sight))

S15 TX ((handicap* $n 3$ vision) or (handicap* n3 visual* ${ }^{\star}$ or (handicap* n3 sight))

S14 TX ((low* $n 3$ vision) or (low ${ }^{\star} n 3$ visual $\left.{ }^{\star}\right)$ or (low ${ }^{\star} n 3$ sight $)$ )

S13 (MH "Vision Disorders+")

$\mathrm{S} 12 \mathrm{~S} 1$ or $\mathrm{S} 2$ or $\mathrm{S} 3$ or $\mathrm{S} 4$ or $\mathrm{S} 5$ or $\mathrm{S} 6$ or $\mathrm{S} 7$ or $\mathrm{S} 8$ or $\mathrm{S} 9$ or $\mathrm{S} 10$ or $\mathrm{S} 11$

S11 TX allocat* random* $^{\star}$

S10 (MM "Quantitative Studies")

S9 (MM "Placebos")

S8 TX placebo*

S7 TX random* allocat*

S6 (MM "Random Assignment")

S5 TX randomi ${ }^{\star}$ control $^{\star}$ trial $^{\star}$

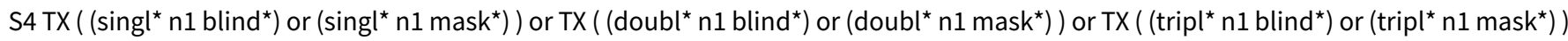
or TX ( (trebl* $n 1$ blind $\left.^{\star}\right)$ or (trebl* $n 1$ mask $\left.\left.^{\star}\right)\right)$

S3 TX clinic $^{*}$ n1 trial ${ }^{\star}$

S2 PT Clinical trial

S1 (MH "Clinical Trials+")

\section{Appendix 5. AMED OvidSP search strategy}

1. vision disorders/

2. ((low\$ or handicap\$ or subnormal\$ or impair\$ or partial\$ or disab\$) adj3 (vision or visual\$ or sight\$)).tw.

3. or $/ 1-2$

4. Rehabilitation/

5. ((rehabilitat\$ or assess\$) adj4 low vision).tw.

6. "Activities of daily living"/

7. Risk/

8. Safety/

9. (home adj3 safety\$).tw.

10. (hazard\$ adj3 (home or environment\$)).tw.

11. Home care services/

12. Occupational therapy/

13. Exercise therapy/

14. physical therapy modalities/

15. (behavio $\$$ adj3 modif\$).tw.

16. (program $\$$ adj3 (home or exercise $\$$ or modif $\$)$ ).tw.

17. Cognitive therapy/

18. Behavior therapy/

19. or/4-18

20. 3 and 19

21. "Randomized controlled trials"/

Environmental and behavioural interventions for reducing physical activity limitation and preventing falls in older people with visual 
22. prospective studies/

23. single blind method/

24. random\$.tw.

25. placebo $\$ . t w$.

26. trial\$.tw.

27. groups.tw.

28. ((singl\$ or doubl\$) adj3 (blind\$ or mask\$)).tw.

29. or/21-28

30.20 and 29

\section{Appendix 6. OTseeker search strategy}

low vision AND rehabilitation AND random

\section{Appendix 7. metaRegister of Controlled Trials search strategy}

low vision and rehabilitation

\section{Appendix 8. ISRCTN search strategy}

("low vision" OR "visual impairment") AND (rehabilitation OR behavioural)

\section{Appendix 9. ClinicalTrials.gov search strategy}

(low vision OR visual impairment) AND (rehabilitation OR behavioural)

\section{Appendix 10. WHO ICTRP search strategy}

low vision OR visual impairment AND rehabilitation OR behavioural

\section{Appendix 11. List of abbreviations}

Phone-FITT: Telephone questionnaire for self-report of physical activity

SFES-I: Short Falls Efficacy Scale-International

EuroQoL: European Quality of Life

GDS: Geriatric Depression Scale

PANAS: Positive and Negative Affect Scale

O\&M: Orientation and Mobility

PASE: Physical Activity Scale for the Elderly

SF-12: 12-Item Short Form Health Survey

SD-36: 36-Item Short Form Health Survey

AFRIS: Attitudes to Falls-Related Interventions Scale

FABQ: Fear-Avoidance Beliefs Questionnaire

\section{WHAT'S NEW}

\begin{tabular}{lll}
\hline Date & Event & Description \\
\hline 20 August 2020 & $\begin{array}{l}\text { New citation required and conclusions } \\
\text { have changed }\end{array}$ & $\begin{array}{l}\text { Issue 8 2020: The update of this review has led to changes in the } \\
\text { conclusions. }\end{array}$ \\
\hline 20 August 2020 & New search has been performed & $\begin{array}{l}\text { Issue 8 2020: Updated searches completed in February 2020 } \\
\text { yielded six studies for inclusion. The previous published version } \\
\text { of this review had no included studies (Skelton 2013). }\end{array}$ \\
\hline
\end{tabular}

Environmental and behavioural interventions for reducing physical activity limitation and preventing falls in older people with visual 


\section{HIST O R Y}

Protocol first published: Issue 7, 2011

Review first published: Issue 6, 2013

\section{CONTRIBUTIONS OF AUTHORS}

\section{Authors contributing to this update review:}

Conceiving the update review: JE, DS, TL

Designing the update review: JE, DS, TL

Screening search results: JE, US, LM, KT, LG, TH

Extracting data: JE, DS, KT, TH

Writing the first draft: JE, DS

Revising the review: JE, DS, TL

Commenting on the review: JE, DS, TL, US, LM, KT, LG, TH

Previous version of the review (2013): Skelton, Ballinger, Neil and Howe conceived and developed the protocol. Gray and Palmer commented on drafts of the protocol and search strategies. Skelton and Howe reviewed all selected publications, with Gray as adjudicator. Ballinger, Howe, Palmer and Gray commented on drafts of the review.

\section{DECLARATIONS OF INTEREST}

DS is a Director of Later Life Training Ltd, a not-for-profit training company in the UK that delivers training to health and fitness professionals in delivery of the Fall Management Exercise and Otago exercise programs. JE, TL, LM, KT, US, LG, and TH have no conflicts of interest to disclose.

\section{SOURCES OF SUPPORT}

\section{Internal sources}

- Glasgow Caledonian University, UK

Salary DS, LG, US, KT

- National Institutes of Health: UG1 EY020522, USA

Salary JE, TJ

\section{External sources}

- National Institute for Health Research (NIHR), UK

* Richard Wormald, Co-ordinating Editor for Cochrane Eyes and Vision (CEV) received financial support for his CEV research sessions (during this update) from the Department of Health through the award made by the National Institute for Health Research to Moorfields Eye Hospital NHS Foundation Trust and UCL Institute of Ophthalmology for a Specialist Biomedical Research Centre for Ophthalmology.

* This review update was supported by the NIHR, via Cochrane Infrastructure funding to the CEV UK editorial base.

The views and opinions expressed therein are those of the authors and do not necessarily reflect those of the Systematic Reviews Programme, NIHR, NHS or the Department of Health.

\section{DIFFERENCES BETWEEN PROTOCOLANDREVIEW}

\section{0 review update}

- The title and objectives have been changed for the update. "Preventing falls" was added into the title. The population qualifier "community-dwelling" has been removed from the title of this review since the last version was published, but the inclusion criteria with respect to the study population have not changed, i.e. we included older people living independently and those living in residential settings.

- We changed falls from a secondary to a primary outcome of this review, because there is no specific review to study the effect of environmental and behavioral interventions to reduce falls in visually impaired older adults. We deemed reducing physical activity limitation to be a relevant outcome in fall prevention trials. To be eligible for inclusion in this update, studies had to report physical activity and/or fall-related measures.

- Five new authors joined the review team: JE, TL, LM, KT, US.

Environmental and behavioural interventions for reducing physical activity limitation and preventing falls in older people with visual 


\section{NOTES}

None

\section{NDEX TERMS}

\section{Medical Subject Headings (MeSH)}

*Independent Living; *Motor Activity; Visually Impaired Persons [`rehabilitation]

\section{MeSH check words}

Aged; Humans 